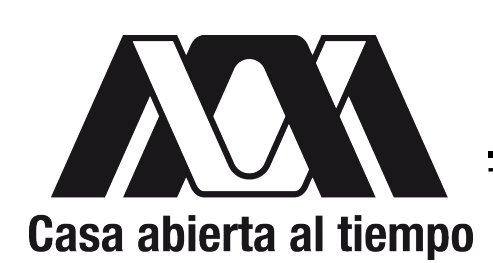

Casa abierta al tiempo

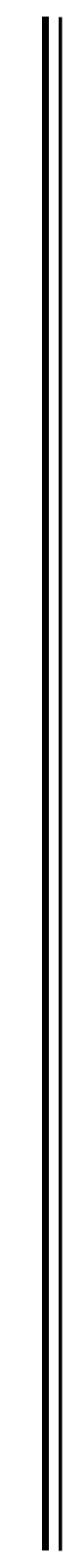

UNIVERSIDAD AUTÓNOMA METROPOLITANA

DIVISIÓN DE CIENCIAS BÁSICAS E INGENIERÍA UNIDAD IZTAPALAPA

DEPARTAMENTO DE MATEMÁTICAS

\section{MAXIMALIDAD DE PROPIEDADES \\ QUE GENERALIZAN LA COMPACIDAD}

Tesis que para obtener el grado de

DOCTOR EN CIENCIAS (MATEMÁTICAS)

presenta

M. EN C. JUAN ALBERTO MARTÍNEZ CADENA

Directores de tesis:

DR. RICHARD G. WILSON

DR. MANUEL SANCHIS LÓPEZ

Sinodales:

Presidente: DR. RICHARD G. WILSON

Secretario: DR. VLADIMIR TKACHUK

Vocal: DR. SALVADOR GARCÍA FERREIRA

Vocal: DR. ÁNGEL TAMARIZ MASCARÚA

Vocal: DR. MANUEL SANCHIS LÓPEZ

Ciudad de México

5 de noviembre de 2018 



\section{Índice general}

$\begin{array}{ll}\text { Introducción } & 1\end{array}$

1. Preliminares 3

1.1. Notación y terminología . . . . . . . . . . . . . . . . 3

1.2. Propiedades Básicas . . . . . . . . . . . . . . . . 4

1.3. Propiedades Básicas de Grupos

Topológicos y Paratopológicos . . . . . . . . . . . . . 6

2. Espacios Densamente Numerablemente Compactos 9

2.1. Topologías maximales Densamente

Numerablemente Compactas . . . . . . . . . . . . . . . . 11

2.1.1. Espacios T-maximales Densamente

Numerablemente Compactos . . . . . . . . . . . . . 21

2.2. Grupos Topológicos y Paratopológicos

Densamente Numerablemente Compactos . . . . . . . . . . 23

3. Espacios Selectivamente Tenuemente Compactos 27

3.1. Espacios Maximales Selectivamente

Tenuemente Compactos . . . . . . . . . . . . . . . . 32

3.1.1. Espacios T-maximales Selectivamente

Pseudocompactos . . . . . . . . . . . . . 35

3.2. Grupos Topológicos y Paratopológicos

Selectivamente Tenuemente Compactos . . . . . . . . 36

4. Espacios Secuencialmente Tenuemente Compactos 39

4.1. Espacios Maximales Secuencialmente

Tenuemente Compactos . . . . . . . . . . . . . . . . 43 
4.1.1. Espacios T-maximales Secuencialmente

Pseudocompactos . . . . . . . . . . . . . . 45

4.2. Grupos Topológicos Secuencialmente

Tenuemente Compactos . . . . . . . . . . . . . . . . . 47

5. Conclusiones 49

5.1. Preguntas abiertas ................... . . 49 


\section{Introducción}

Las propiedades topológicas que se encuentran entre las propiedades de compacidad numerable y pseudocompacidad son de gran interés tanto en la topología general y en el álgebra topológica siendo fuertemente estudiadas en los últimos años.

El objetivo principal del presente trabajo es presentar los resultados obtenidos sobre las propiedades maximales en la clase de espacios topológicos (y en la clase de espacios de Tychonoff) de las propiedades topológicas que se encuentran entre las clases de compacidad numerable y compacidad tenue, tales como la propiedad Densamente Numerablemente Compacta y la propie-

dad Selectivamente Tenuemente Compacta. Además, mostrar resultados sobre los espacios Secuencialmente Tenuemente Compactos que son más generales que los espacios secuencialmente compactos y cuyas propiedades topológicas resultan de importancia en la clase de los espacios tenuemente compactos.

En 1947, Vaidyanathaswamy mostró que la topología de todo espacio compacto de Hausdorff es maximal compacta ([44]) en la clase de todos los espacios topológicos. En 1948, A. Ramanathan ([37]) caracteriza las topologías maximales compactas como aquellas cuyos subconjuntos compactos son cerrados. Además, exhibe un espacio maximal compacto que no es Hausdorff.

Al hacer un estudio de las propiedades maximales de topologías numerablemente compactas y pseudocompactas encontramos algunos hechos interesantes. A. B. Raha prueba que un espacio numerablemente compacto $X$ es maximal numerablemente compacto si y sólo si todos los subconjuntos numerablemente compactos de $X$ son cerrados (Teorema 4 en [36]). Las topologías maximales tenuemente compactas fueron rigurosamente estudiadas en la clase de todos los espacios topológicos por J. R. Porter, R. M. Stephenson y R. G. Woods en [33] y [34]. También, caracterizan a los espacios maximal tenuemente compactos como aquellos que son tenuemente compactos, submaximales y donde todos sus subconjuntos tenuemente compactos son cerrados. 
En los últimos años las propiedades maximales de los espacios numerablemente compactos y pseudocompactos fueron estudiadas en la clase de los espacios de Tychonoff por O. T. Alas, M. Sanchis, V. V. Tkachuk y R. G. Wilson en [3], [6] y [42]. Recientemente, las propiedades maximales de los espacios densamente numerablemente compactos, selectivamente compactos y secuencialmente compactos fueron estudiadas en la clase de los espacios topológicos y en la clase de los espacios de Tychonoff por O.T. Alas, J. A. Martínez-Cadena y R. G. Wilson en [1] y [27].

El siguiente trabajo se encuentra dividido en tres capítulos, en los cuales se hace un estudio de cada una de las propiedades topológicas que generalizan la compacidad y que son de nuestro interes, al final de cada capítulo mostraremos algunos resultados obtenidos sobre estas propiedades en la clase de grupos topológicos y paratopológicos.

En el primer capítulo nos enfocaremos en el estudio de las propiedades maximales de los espacios densamente numerablemente compactos $(D N C)$. Mostraremos una caracterización de los espacios maximales $D N C$ (Teorema 2.13) y daremos condiciones bajo las cuales la topología de un espacio $D N C$ tiene un refinamiento maximal $D N C$ (Teorema 2.20), así como una condición suficiente para que un espacio $D N C$ de Tychonoff sea T-maximal $D N C$ (Corolario 2.29).

En el segundo capítulo estudiaremos las propiedades maximales de los espacios selectivamente tenuemente compactos (SelTC), concluyendo con una caracterización de los espacios maximales SelTC (Corolario 3.14). También, daremos condiciones bajo las cuales la extensión de Katětov $\kappa X$ de un espacio de Hausdorff $X$ resulta ser un espacio selectivamente tenuemente compacto (Teorema 3.6).

Finalmente, en el tercer capítulo realizaremos un estudio sobre algunas extensiones de los espacios secuencialmente tenuemente compactos (SecTC), presentando resultados sobre la extensión de Katětov (Teorema 4.3), la compactificación en un punto (Teorema 4.4) y la compactificación de Stone-Čech (Teorema 4.6), así como una caracterización de espacios maximales SecTC (Corolario 4.8). 


\section{Capítulo 1}

\section{Preliminares}

Este capítulo está diseñado con la idea de que el lector pueda revisar en él algunos conceptos básicos de la teoría de espacios topológicos y grupos topológicos, los cuales serán de gran utilidad para la comprensión de los resultados que se mostrarán en los siguientes capítulos. Sin embargo, debemos de señalar que en este apartado no se hallará un desarrollo detallado de los distintos temas que se tocan en el mismo y que algunas definiciones necesarias en este estudio, se irán presentando a lo largo del texto.

\subsection{Notación y terminología}

Un espacio topológico es tenuemente compacto si toda familia localmente finita de subconjuntos abiertos no vacíos es finita, ó equivalentemente, si toda familia infinita de subconjuntos abiertos no vacíos tiene un punto de acumulación. Un espacio topológico $X$ es llamado pseudocompacto si toda función continua con dominio $X$ y valores reales es acotada. Este concepto fue introducido por E. Hewitt en [23].

Es conocido el hecho de que en la clase de espacios de Tychonoff las propiedades de ser tenuemente compacto y pseudocompacto resultan ser equivalentes. De aquí en adelante, los espacios pseudocompactos serán asumidos de Tychonoff y los espacios tenuemente compactos serán asumidos por lo menos $T_{1}$.

Notemos que si existe una familia infinita $\mathcal{F}$ localmente finita de subconjuntos abiertos no vacíos, entonces existe una familia infinita $\mathcal{G}$ localmente finita de subconjuntos abiertos no vacíos ajenos dos a dos con la propiedad 
de que cada elemento de $\mathcal{G}$ está contenido en algún elemento distinto de $\mathcal{F}$. Por lo tanto, si existe una familia infinita localmente finita de subconjuntos abiertos no vacíos en un espacio $X$, entonces también existe una familia infinita localmente finita de subconjuntos abiertos no vacíos ajenos dos a dos. Por lo cual, para probar que un espacio $X$ es tenuemente compacto, es suficiente probar que toda familia infinita de subconjuntos abiertos no vacíos ajenos dos a dos en $X$, tiene un punto de acumulación en $X$.

Para un espacio topológico $X$, denotaremos por $w(X), d(X), \chi(X), \psi(X)$, $e(X)$ y $c(X)$ el peso, densidad, carácter, pseudocarácter, extensión y celularidad respectivamente. El $\pi$-peso y el $\pi$-carácter de $X$ son denotados por $\pi w(X)$ y $\pi \chi(X)$.

La clausura de un subconjunto de un espacio topológico $(X, \tau)$ será denotada por $c l_{\tau}(A), c l_{X}(A)$ ó si ninguna confusión es posible, simplemente por $c l(A)$, similarmente $\operatorname{int}_{\tau}(A), \operatorname{int}_{X}(A)$ ó simplemente $\operatorname{int}(A)$ denota el interior de $A$ en $(X, \tau)$. A menos que se indique lo contrario, una sucesión es siempre indexada por $\omega$ y no se asume inyectiva. Aunque el rango de una sucesión de conjuntos no vacíos ajenos disjuntos es una familia infinita numerable de conjuntos, optaremos por utilizar el término sucesión excepto cuando nos refieramos a familias localmente finitas y discretas. En un espacio topológico, una sucesión $\left\langle U_{n}\right\rangle$ de conjuntos abiertos converge a $p$ (escribiremos $\left\langle U_{n}\right\rangle \rightarrow p$ ), si toda vecindad de $p$ contiene a todos excepto un número finito de elementos de la sucesión.

Todos los términos indefinidos se pueden encontrar en [8], [18] y [24].

\subsection{Propiedades Básicas}

Sean $\mathcal{C}$ una clase de espacios topológicos y $\mathcal{P}$ una propiedad topológica; un espacio $X$ en la clase $\mathcal{C}$ es llamado maximal $\mathcal{P}$ en $\mathcal{C}$ si $X$ tiene $\mathcal{P}$ y ninguna otra topología más fuerte en $\mathcal{C}$ sobre $X$ tiene $\mathcal{P}$. Si $\mathcal{C}$ es la clase de todos los espacios topológicos, entonces diremos que la topología es universalmente maximal $\mathcal{P}$ y si ninguna confusión es posible, diremos que la topología es maximal $\mathcal{P}$.

Si $(X, \tau)$ es un espacio topológico y $\mathcal{F}$ es un filtro maximal en la familia de subconjuntos densos de $X$, entonces la topología $\sigma$ generada por la subbase $\tau \cup \mathcal{F}$ es llamada una submaximalización de $\tau$ y $(X, \sigma)$ es un espacio submaximal, es decir, un espacio donde todo subconjunto denso es abierto. 
Notemos que la submaximalización de un espacio tenuemente compacto es tenuemente compacto. Por lo tanto, la topología de un espacio maximal tenuemente compacto debe ser submaximal (Teorema 14 en [36]).

Dado un espacio $(X, \tau)$, denotamos por $\tau_{S}$ la topología en $X$, cuya base consiste de los subconjuntos abiertos regulares de $(X, \tau)$. Diremos que una propiedad topológica $\mathcal{P}$ es semiregular si para un espacio topológico $(X, \tau)$ se cumple: $(X, \tau)$ tiene $\mathcal{P}$ si y sólo si $\left(X, \tau_{S}\right)$ tiene $\mathcal{P}$.

Sea $X$ un espacio topológico y $\mathcal{P}$ una propiedad topológica. Consideremos las siguientes propiedades:

i) $\mathcal{P}$ se preserva bajo funciones continuas.

ii) $\mathcal{P}$ se hereda a subespacios cerrados regulares.

iii) $\mathcal{P}$ es semiregular.

iv) Si $D$ es un espacio denso de $X$ que tiene $\mathcal{P}$ entonces $X$ tiene $\mathcal{P}$.

v) $\mathcal{P}$ se preserva bajo uniones finitas de espacios topológicos.

Los siguientes cuatro hechos fueron mostrados por D. Cameron en [12]:

Teorema 1.1 ([12]). Si la propiedad topológica $\mathcal{P}$ satisface iii), entonces cualquier topología maximal $\mathcal{P}$ es submaximal.

Teorema 1.2 ([12]). Sea $X$ un espacio submaximal. Si $X$ con la propiedad $\mathcal{P}$ satisface $i)-v$ ), entonces $X$ es maximal $\mathcal{P}$ si y sólo si para todo $A \subset X$ tal que $A$ y $X \backslash \operatorname{int}(A)$ satisfacen $\mathcal{P}$, entonces $A$ es cerrado.

Corolario 1.3. Sea $X$ un espacio submaximal con la propiedad $\mathcal{P}$ que satisface i)-v). Si todo subespacio con la propiedad $\mathcal{P}$ es cerrado, entonces $X$ es maximal $\mathcal{P}$.

Teorema 1.4 ([12]). Si la propiedad $\mathcal{P}$ satisface i)-v) y todos los conjuntos unitarios tienen la propiedad $\mathcal{P}$, entonces los espacios maximal $\mathcal{P}$ son $T_{1}$.

Por los resultados anteriores y la parte a) $\rightarrow$ b) del Teorema 2.2 en [33], se obtiene:

Teorema 1.5 ([33]). Un espacio topológico es maximal tenuemente compacto si y sólo si $X$ es submaximal y todos sus subespacios tenuemente compactos de $X$ son cerrados. 
A. B. Raha muestra la siguiente caracterización para espacios maximales numerablemente compactos (Teorema 4 en [36]):

Teorema 1.6 ([36]). Un espacio topológico es maximal numerablemente compacto si y sólo si todos sus subespacios numerablemente compactos son cerrados.

\subsection{Propiedades Básicas de Grupos Topológicos y Paratopológicos}

Un grupo $G$ dotado con una topología $\tau$ es llamado grupo paratopológico si la multiplicación en $G$ definida como un mapeo de $G \times G$ a $G$ es continua, donde $G \times G$ tiene la topología producto. Un grupo topológico es un grupo paratopológico donde la inversion en $G$ definida como $\operatorname{In}(x)=x^{-1}$ es continua.

Dado un grupo $G$ y un elemento $a \in G$, definimos las traslaciones $\lambda_{a}$ y $\varrho_{a}$ (izquierda y derecha respectivamente) como

$$
\lambda_{a}(x)=a x \quad \mathrm{y} \quad \varrho_{a}(x)=x a,
$$

para cada $x \in G$. Observemos que $\lambda_{a}$ y $\varrho_{a}$ son biyecciones de $G$ sobre sí mismo.

Teorema 1.7 ([8]). Sea $G$ un grupo paratopológico y $\mathcal{U}$ una base local del neutro e en $G$. Entonces:

i) para $U, V \in \mathcal{U}$, existe $W \in \mathcal{U}$, tal que $W \subset U \cap V$;

ii) para cada $U \in \mathcal{U}$ y cada $x \in U$, existe $V \in \mathcal{U}$ tal que $V x \subset U$;

iii) para cada $U \in \mathcal{U}$ y cada $x \in G$, existe $V \in \mathcal{U}$ tal que $x V x^{-1} \subset U$;

iv) para cada $U \in \mathcal{U}$, existe $V \in \mathcal{U}$ tal que $V^{2} \subset U$.

Reciprocamente, sea $G$ un grupo y sea $\mathcal{U}$ la familia de subconjuntos de $G$ que satisface las condiciones $i)$-iv). Entonces la familia $\mathcal{B}_{\mathcal{U}}=\{U a: a \in$ $G, U \in \mathcal{U}\}$ es una base para la topología $\tau_{\mathcal{U}}$ en $G$ tal que $\left(G, \tau_{\mathcal{U}}\right)$ es un grupo paratopológico. Además, la familia $\{a U: a \in G, U \in \mathcal{U}\}$ es una base para la misma topología $\tau_{\mathcal{U}}$. 
Demostración. Supongamos que $G$ es un grupo paratopológico. La parte i) es válida porque $\mathcal{U}$ es una base local del neutro $e$ de $G$. Los incisos ii) y iii) se siguen de la continuidad de las funciones $\lambda_{x}$ y $\lambda_{x^{-1}} \circ \varrho_{x}$. Como la multiplicación es continua en $G$, se satisface iv).

Probemos el recíproco. Sea $\mathcal{U}$ una familia de subconjuntos de $G$ que satisface las condiciones i)-iv). Sea $\tau$ la familia de conjuntos $W \subset G$ que satisfacen la siguiente condición:

(*) para cada $x \in W$, existe $U \in \mathcal{U}$ tal que $U x \subset W$.

Afirmación 1: $\tau$ es una topología en $G$.

Notemos que el vacío y $G$ están en $\tau$. También, que la unión arbitraria de elementos de $\tau$ está en $\tau$. Ahora, sean $W_{1}, W_{2} \in \tau$. Sea $W=W_{1} \cap W_{2}$. Tomemos $x \in W$, existen $U_{1}, U_{2} \in \mathcal{U}$ tales que $U_{1} x \subset W_{1}$ y $U_{2} x \subset W_{2}$. Por i), existe $U \in \mathcal{U}$ tal que $U \subset U_{1} \cap U_{2}$. Por lo tanto, $U x \subset W_{1} \cap W_{2}=W$. Se sigue que $W \in \tau$. Hemos probado la afirmación.

Afirmación 2: $U x \in \tau$, para cada $x \in G$ y cada $U \in \mathcal{U}$.

Tomemos $y \in U x$, equivalentemente, $y x^{-1} \in U$. Por ii), existe $V \in \mathcal{U}$ el cual satisface $V y x^{-1} \subset U$, es decir, $V y \subset U x$. Por lo tanto, el conjunto $U x$ está en $\tau$.

La afirmación 2 y la condición ii) implican lo siguiente:

Afirmación 3: La familia $\mathcal{B}_{\mathcal{U}}=\{U a: a \in G, U \in \mathcal{U}\}$ es una base para la topología $\tau$. En consecuencia $\tau=\tau_{\mathcal{U}}$.

Afirmación 4: La multiplicación en $G$ es continua respecto a la topología $\tau$.

Sean $a, b \in G$ y $O$ un elemento de $\tau$ tal que $a b \in O$. Por definición de $\tau$, podemos encontrar $W \in \mathcal{U}$ tal que $W a b \subset O$. La condición iv) garantiza la existencia de un elemento $U \in \mathcal{U}$ que satisface $U^{2} \subset W$. Por iii), existe $V \in \mathcal{U}$ tal que $a V a^{-1} \subset U$. Se sigue de lo anterior que $U\left(a V a^{-1}\right) \subset U^{2} \subset W$, por lo tanto, $U a V b=U\left(a V a^{-1}\right) a b \subset W a b \subset O$. La afirmación está probada.

Afirmación 5: $b V \in \tau$, para cada $b \in G$ y cada $V \in \mathcal{U}$.

Tomemos $y \in b V$, es decir, $b^{-1} y \in V$. Por ii), existe $W \in \mathcal{U}$ tal que $W b^{-1} y \subset V$. Aplicando iii), podemos encontrar un elemento $U \in \mathcal{U}$ el cual satisface $b^{-1} U b \subset W$. En consecuencia, $b^{-1} U y=\left(b^{-1} U b\right) b^{-1} y \subset W b^{-1} y \subset V$, esto es, $b^{-1} U y \subset V$. Por lo tanto, $U y \subset b V$. Hemos probado la afirmación.

Por último, de la afirmación 5 y la condición iii) se concluye que la familia $\{a U: a \in G, U \in \mathcal{U}\}$ también es una base para la topología $\tau$. 
Si $A$ es un subconjunto de un grupo semitopológico $G$, definimos el inverso de $A$ como $A^{-1}=\left\{a^{-1}: a \in A\right\}$. Y decimos que $A$ es simétrico si $A=A^{-1}$.

Proposición 1.8. Sea $G$ un grupo paratopológico y $\mathcal{U}$ una base local del neutro en $G$. Entonces son equivalentes:

a) $G$ es un grupo topológico;

b) para cada $U \in \mathcal{U}$, existe $V \in \mathcal{U}$ tal que $V^{-1} \subset U$.

Demostración. a) implica b) porque la inversión es continua en la identidad. Probemos que b) implica a). Tomemos un abierto $O$ en $G$ y un elemento arbitrario $o \in O$. El conjunto $o^{-1} O$ es una vecindad abierta de $e$. Como $\mathcal{U}$ es una base local de $e$, podemos encontrar $U \in \mathcal{U}$ tal que $U \subset o^{-1} O$. Por hipótesis, existe $V \in \mathcal{U}$ el cual satisface $V^{-1} \subset U$. Por lo tanto, $V \subset U^{-1} \subset$ $O^{-1} o$, en consecuencia, $o^{-1} \in V o^{-1} \subset O^{-1}$. Como $G$ es grupo paratopológico, $V o^{-1}$ es una vecindad abierta de $O^{-1}$. Por lo tanto, el conjunto $O^{-1}$ es abierto en $G$. Hemos demostrado que la inversión es continua en $G$.

Proposición 1.9. Todo grupo topológico G tiene una base local en el neutro e, que consiste de vecindades abiertas simétricas.

Demostración. Para un vecindad abierta $U$ en $e$, sea $V=U \cap U^{-1}$. Entonces $V=V^{-1}$, y el conjunto $V$ es una vecindad abierta de $e$ con $V \subset U$.

Dado un grupo paratopológico $G, \xi(e)$ denotará el conjunto de vecindades abiertas del elemento neutro $e$ en $G$.

Sea $G$ un grupo paratopológico dotado con una topología $\tau$. Definimos la topología conjugada $\tau^{-1}$ en $G$ como

$$
\tau^{-1}=\left\{U^{-1}: U \in \tau\right\} .
$$

Esta topología hace que $\left(G, \tau^{-1}\right)$ sea un grupo paratopológico. Es inmediato que $(G, \tau)$ y $\left(G, \tau^{-1}\right)$ son homeomorfos. El límite superior $\tau^{*}=\tau \vee \tau^{-1}$ es una topología de grupo en $G$ y $G^{*}=\left(G, \tau^{*}\right)$ es el grupo topológico asociado al grupo paratopológico $(G, \tau)$. Notemos que $\tau^{*}$ es la mínima de las topologías de grupo en $G$ que contienen a $\tau$.

Si $G$ es un grupo paratopológico con topología $\tau$, entonces la diagonal $\Delta_{G}=\{(x, x): x \in G\}$ con la topología que hereda del producto $(G, \tau) \times$ $\left(G, \tau^{-1}\right)$ es un grupo topológico y es topológicamente isomorfo al grupo topológico asociado $G^{*}$ de $G$. 


\section{Capítulo 2}

\section{Espacios Densamente Numerablemente Compactos}

Un espacio topológico $X$ se dice que es densamente numerablemente compacto, si éste posee un subespacio denso $D$ con la propiedad de que cualquier subconjunto infinito de $D$ tiene un punto de acumulación en $X$. Este concepto fue introducido en 1981 por A. Berner en [10] bajo el nombre de espacios con un subconjunto denso condicionalmente compacto, también son conocidos como espacios numerablemente pracompactos en [39]. Debido a la longitud del nombre de estos espacios, en ocaciones los espacios densamente numerablemente compactos serán denotados como $\boldsymbol{D N C}$, principalmente en las demostraciones de los resultados a lo largo del texto.

Notemos que si $X$ es un espacio $D N C$ y $D$ es testigo de este hecho, entonces toda sucesión de abiertos ajenos dos a dos en $X$ contiene un punto de $D$ y la colección de todos estos puntos forman una sucesión que tiene un punto de acumulación $x \in X$. Por lo cual, se puede concluir que $x$ también es punto de acumulación de la sucesión de conjuntos abiertos, esto es, todo espacio $D N C$ es tenuemente compacto. También, por el Teorema 3.10.3 en [18], tenemos que todo espacio numerablemente compacto es $D N C$.

Una colección $\mathcal{A}$ de subconjuntos infinitos de $\omega$ es una familia casiajena si para todo $A, B \in \mathcal{A}$ se tiene que $A \cap B$ es finita. El siguiente ejemplo apareció por primera vez en [30]:

Ejemplo 2.1 (Espacio de Mrówka). Existe un espacio de Tychonoff localmente compacto, primero numerable y densamente numerablemente compacto que no es numerablemente compacto. 
Demostración. Sea $\mathcal{A}$ una familia infinita maximal casi-ajena de subconjuntos de $\omega$, definimos el espacio $\Psi(\mathcal{A})=\mathcal{A} \cup \omega$, con la topología:

1) Los puntos en $\omega$ son aislados,

2) Para $A \in \mathcal{A}$, un abierto básico es de la forma $\{A\} \cup(A \backslash F)$, donde $F \subset \omega$ es finito.

Una prueba detallada de las propiedades de $\Psi(\mathcal{A})$ pueden encontrarse en [21]. Notemos que $\omega$ es un conjunto denso de puntos aislados de $\Psi(\mathcal{A})$ que cumple la definición de espacio $D N C$ y también, $\mathcal{A}$ es un conjunto infinito, cerrado y discreto de $\Psi(\mathcal{A})$, por lo tanto $\Psi(\mathcal{A})$ no es numerablemente compacto.

A. Berner muestra el siguiente ejemplo, del cual sólo presentamos una breve idea de la construcción :

Ejemplo 2.2 ([10]). Existe un espacio localmente compacto, primero numerable y pseudocompacto que no es densamente numerablemente compacto.

Demostración. Denotamos como $K$ el conjunto ternario de Cantor en $\mathbb{R}$. Si $\left\langle U_{n}\right\rangle$ es una sucesión de conjuntos abiertos no vacíos en $K$, entonces existe un subconjunto infinito $I \subset \omega$ y una sucesión $\left\langle V_{n}\right\rangle_{n \in I}$ de conjuntos abiertos no vacíos ajenos dos a dos en $K$ tal que $V_{n} \subset U_{n}$, para cada $n \in I$.

Sea $D\left(\mathfrak{c}^{+}\right)$un espacio discreto de cardinalidad $\mathfrak{c}^{+}$y sea $N=K \times D\left(\mathfrak{c}^{+}\right)$. Supongamos que $\left\langle U_{i} \times\left\{\alpha_{i}\right\}\right\rangle_{i \in \omega}$ y $\left\langle U_{j} \times\left\{\beta_{j}\right\}\right\rangle_{j \in \omega}$ son sucesiones de abiertos básicos en $N$. Diremos que estas sucesiones son casi-ajenas si $\left\langle U_{i} \times\left\{\alpha_{i}\right\}\right\rangle_{i \in \omega} \cap$ $\left\langle U_{j} \times\left\{\beta_{j}\right\}\right\rangle_{j \in \omega}=\emptyset$ para todo excepto un número finito de parejas $(i, j)$.

Sea $S$ una colección maximal de sucesiones casi-ajenas $\left\langle U_{i} \times\left\{\alpha_{i}\right\}\right\rangle_{i \in \omega}$ de abiertos básicos en $N$ que cumplen:

1) $i \neq j$, entonces $\alpha_{i} \neq \alpha_{j} \mathrm{y}$

2) $i \neq j$, entonces $U_{i} \cap U_{j}=\emptyset$.

Para cada sucesión $s \in S$, tomamos un punto $p_{s}$, donde un abierto básico de $p_{s}$ es de la forma $\left\{p_{s}\right\} \cup \bigcup\left\{U_{i} \times\left\{\alpha_{i}\right\}: i>n\right\}$ para algún $n \in \omega$. Sea $L=\left\{p_{s}: s \in S\right\}$ y denotamos $X=N \cup L$.

El espacio $X$ es el espacio que cumple las propiedades requeridas del ejemplo (para una prueba detallada de las propiedades de este espacio vease el Ejemplo 5 en [10]).

Por los Ejemplos 2.1 y 2.2, concluimos que la clase de los espacios $D N C$ se encuentra entre la clase de los espacios numerablemente compactos y la clase de los espacios pseudocompactos de manera estricta. 
Teorema 2.3. Sea $X$ un espacio topológico y $D$ un subconjunto denso de puntos aislados de $X$. Entonces, $X$ es tenuemente compacto si y sólo si es densamente numerablemente compacto.

Demostración. Bastará probar la suficiencia. Supongamos que $X$ no es $D N C$, es decir, existe un subconjunto infinito $A \subset D$ sin punto de acumulación, por lo cual $A$ es cerrado en $X$. Por otra parte, la familia de puntos de $A$ es una familia infinita de conjuntos abiertos ajenos dos a dos sin punto de acumulación y esto contradice la compacidad tenue.

\subsection{Topologías maximales Densamente Numerablemente Compactas}

Comenzamos esta sección, observando que un espacio topológico submaximal y denso en sí es $T_{1}$, esto es, si $X$ es un espacio topológico con dichas propiedades, entonces para algún $x \in X$, el espacio $X \backslash\{x\}$ es denso y por lo tanto abierto. Esto es, el conjunto unitario $\{x\}$ es cerrado.

$\mathrm{Si}$ un subconjunto $A$ de un espacio topológico $X$ no contiene puntos aislados, se llamará denso en si. Es conocido el hecho de que un espacio infinito de Hausdorff contiene un subconjunto infinito discreto ([18]); por lo cual un espacio infinito de Hausdorff submaximal denso en sí contiene un subespacio que es cerrado y discreto (por lo tanto denso en sí). Como un subespacio de un espacio submaximal es submaximal, entonces todo subconjunto infinito de un espacio de Hausdorff submaximal y denso en sí contiene un subespacio infinito cerrado y discreto. Esto se puede generalizar a la clase de espacios $T_{1}$.

Teorema 2.4 ([27]). Todo espacio infinito submaximal denso en sí contiene un subconjunto infinito que es cerrado y discreto.

Demostración. Supongamos que $(X, \tau)$ es un espacio submaximal denso en sí (por tanto $T_{1}$ ). Como $X$ es submaximal, es suficiente encontrar un subconjunto de $X$ con interior vacío. Sea $\left\{A_{n}: n \in \omega\right\}$ una partición numerable de $X$ en la cual, cada $A_{n}$ es infinito. Si para algún $k \in \omega, \operatorname{int}_{\tau}\left(A_{k}\right)=\emptyset$, entonces el resultado esta completo. Por otra parte, si cada $A_{n}$ tiene interior no vacío, entonces tomamos $x_{n} \in \operatorname{int}_{\tau}\left(A_{n}\right)$ para cada $n \in \omega$. Debido a que $X$ es denso en sí, el conjunto $\left\{x_{n}: n \in \omega\right\}$ es infinito y tiene interior vacío. 
Corolario 2.5. Sea $X$ un espacio submaximal denso en sí. Entonces, todo subconjunto infinito $A \subset X$ contiene un subconjunto infinito cerrado y discreto en $X$.

Demostración. Supongamos que $A \subset X$ es un subconjunto infinito de $X$. Si $A$ contiene un subconjunto infinito $I$ de puntos aislados en $A$, entonces $I$ es denso en ninguna parte en $X$ y por lo tanto cerrado y discreto en $X$.

Por otra parte, si $A$ contiene un subconjunto infinito $D$ denso en sí, entonces por Teorema 2.4, $D$ contiene un subconjunto infinito con interior vacío en $D$ y por lo tanto en $X$, el cual es cerrado y discreto en $X$.

Un espacio topológico es disperso si todo subconjunto no vacío tiene un punto aislado y diremos que este espacio tiene orden de dispersión 2 si su conjunto de puntos de acumulación es no vacío y discreto.

Un espacio $D N C$ no es necesariamente $T_{1}$. Sea $X=\omega \cup\{\infty\}$ un espacio topológico dotado con la topología $\tau$ generada por la base $\{\{n, \infty\}: n \in$ $\omega\} \cup\{\{\infty\}\}$, entonces el espacio $(X, \tau)$ sólo tiene un punto no cerrado y el conjunto unitario $\{\infty\}$ es denso en $(X, \tau)$. Se sigue que $X$ es $D N C$, pero la topología $\sigma$ sobre $X$ generada por la subbase $\tau \cup\{\omega\}$ hace al espacio $(X, \sigma)$ un espacio infinito y discreto. Esto es, existe un espacio $T_{0}$ con topología $D N C$ tal que ninguna topología $T_{1}$ más fina es $D N C$. Notemos también que $(X, \tau)$ es $T_{0}$, Fréchet y disperso (en el sentido de que todo punto contiene un punto relativamente abierto) con orden de dispersión 2.

Teorema 2.6 ([27]). Todo espacio maximal densamente numerablemente compacto es $T_{1}$.

Demostración. Sean $(X, \tau)$ un espacio $D N C$ y $D$ el subconjunto denso en $X$ testigo de este hecho. Supongamos que existe un punto $p \in X$ tal que $\{p\}$ no es cerrado. Si $p \in X \backslash D$, entonces $D$ testifica nuevamente que $X$ dotado con la topología generada por la subbase $\tau \cup\{X \backslash\{p\}\}$ es un espacio $D N C$. Además, como $X \backslash\{p\} \notin \tau$, entonces la topología generada por la subbase $\tau \cup\{X \backslash\{p\}\}$ es estrictamente más fuerte que $\tau$, lo cual es una contradicción.

Ahora, si $p \in D$, entonces existe algún $q \in c_{\tau}(\{p\}) \backslash\{p\}$ y la topología $\sigma$ sobre $X$, generada por la subbase

$$
\tau \cup\left\{\left(X \backslash c l_{\tau}(\{p\})\right) \cup\{q\}\right\}
$$

es estrictamente más fuerte que $\tau$, ya que $q \notin c l_{\sigma}(\{p\})$. Definimos $D_{\sigma}=$ $\left(D \backslash c l_{\tau}(\{p\})\right) \cup\{p, q\}$. Entonces, tenemos que $D_{\sigma}$ es denso en $(X, \sigma)$ y que 
las topologías $\tau$ y $\sigma$ difieren sólo en $q$. Si $A \subset D_{\sigma}$ es infinito, entonces $A \cap$ $\left(D \backslash c l_{\tau}(\{p\})\right)$ es infinito y se sigue que si $q$ es un $\tau$-punto de acumulación de $A$, entonces $q$ también es un $\sigma$-punto de acumulación de $A$. Por lo tanto, $(X, \sigma)$ es $D N C$.

De aquí en adelante todos los espacios topológicos serán asumidos a lo menos $T_{1}$, por lo cual maximal $D N C$ significará maximal $D N C$ en la clase de espacios $T_{1}$.

Teorema $2.7([27])$. Si $(X, \tau)$ es un espacio maximal densamente numerablemente compacto y $D$ es testigo de este hecho, entonces $D$ es abierto y $X \backslash D$ es cerrado y discreto.

Demostración. Bastará probar que la topología $\sigma$ generada por la subbase

$$
\mathcal{S}=\tau \cup\{D\} \cup\{D \cup\{x\}: x \in X \backslash D\}
$$

es un espacio densamente numerablemente compacto. Con este fin, notemos primero que $D$ es denso en $(X, \sigma)$. Si $A \subset D$ es infinito y $p$ es un punto de acumulación de $A$ en $(X, \tau)$, entonces $p \in D$ ó $p \in X \backslash D$.

Si $p \in D$, como $\sigma|D=\tau| D$, entonces $p$ también es un $\sigma$-punto de acumulación de $A$. Si $p \in X \backslash D$, entonces $p$ es un punto de acumulación de $A$ en $(X, \sigma)$, ya que el filtro de $\tau$-vecindades de $p$ y el filtro de $\sigma$-vecindades de $p$ tiene la misma traza sobre $D$.

Notemos que si $X$ es un espacio $D N C$ y $D$ es el subconjunto denso de $X$ testigo de este hecho, entonces cualquier subconjunto denso de $D$ es denso en $X$ y también es testigo del mismo hecho. Por tanto, se sigue del teorema 2.7 que si $X$ es un espacio maximal $D N C$ y $D$ es el subconjunto denso de $X$ testigo de este hecho, entonces todo subconjunto denso de $D$ debe ser abierto. Por lo cual, podemos obtener el siguiente hecho:

Corolario 2.8. Si $(X, \tau)$ es un espacio maximal densamente numerablemente compacto y $D$ es un subespacio denso de $X$ testigo de este hecho, entonces $(D, \tau \mid D)$ es un espacio submaximal.

Es importante notar que todavia no hemos demostrado que $X$ es submaximal. Supongamos ahora que $(X, \tau)$ es un espacio $D N C$ denso en sí y $D$ es un subespacio denso en $X$ submaximal testigo de este hecho. Por el Corolario 2.5 , sabemos que todo subconjunto infinito de un espacio submaximal denso 
en sí contiene un subconjunto infinito cerrado y discreto, por lo tanto si $A \subset D$ es infinito, entonces $A$ debe tener un punto de acumulación en $X \backslash D$. Reformulamos esto en el siguiente hecho:

Teorema 2.9 ([27]). Supongamos que $(X, \tau)$ es un espacio densamente numerablemente compacto denso en si y $D$ un subconjunto abierto denso submaximal de $X$ testigo de este hecho. Entonces, todo subconjunto infinito $A \subset D$ debe tener un punto de acumulación $p \in X \backslash D$. Más aún, ya sea $A \rightarrow p$ ó $p$ no es el único punto de acumulación de $A$ en $X \backslash D$.

Demostración. Si $A \nrightarrow p$, entonces existe una vecindad $V$ de $p$ en $X$ tal que el conjunto $A \backslash V$ es infinito. Como $A \backslash V \subset A$, entonces $A$ debe tener un punto de acumulación en $X \backslash D$ distinto de $p$.

Teorema 2.10 ([27]). Un espacio maximal densamente numerablemente compacto es un espacio submaximal y disperso.

Demostración. Supongamos que $(X, \tau)$ es un espacio densamente numerablemente compacto y $D$ un subconjunto abierto denso y submaximal de $X$ testigo de este hecho. Definimos $I=\{x \in D:\{x\}$ es abierto $\}$ y $F=$ $D \backslash c l_{\tau}(I)$.

Supongamos que $F \neq \emptyset$, entonces $F$ es un subconjunto abierto denso en sí de $D$ y por tanto de $X$. También, tenemos que cada subconjunto infinito de $I$ tiene un punto de acumulación en $X \backslash(F \cup I)$. Más aún, $G=F \cup I$ es un subconjunto abierto denso de $D$ y por tanto es un subconjunto abierto de $X$ que es testigo de que $X$ es $D N C$ y como $c l(F)$ es un conjunto cerrado regular en $X$, entonces es un espacio $D N C$ (Lema 2.14) y denso en sí. Consideramos la topología $\sigma$ sobre $X$ generada por la subbase:

$$
\mathcal{S}=\tau \cup\{\{x\}: x \in F\} .
$$

Notemos que $(F, \sigma)$ es discreta y por tanto, $\sigma$ es estrictamente más fina que $\tau$. Basta demostrar que $(X, \sigma)$ es un espacio $D N C$. Como $A \subset(G, \sigma)$ es infinito, ya sea $A \cap I$ ó $A \cap F$ es infinito, por Teorema 2.9, $A$ tiene un punto de acumulación en $(X \backslash G, \tau)$ y entonces también en $(X \backslash G, \sigma)$, por lo tanto $(X, \sigma)$ es $D N C$, lo cual es una contradicción.

Por lo anterior, podemos concluir que $F=\emptyset$ y $X$ tiene un conjunto $I$ denso de puntos aislados. Finalmente, del Teorema 2.7 obtenemos que $X \backslash I$ es cerrado y discreto por lo tanto $X$ es disperso de orden de dispersión 2. 
Decimos que un espacio topológico $X$ es un $\boldsymbol{S C}$-espacio si para cualquier sucesión $\left\langle x_{n}\right\rangle$ que converge a $x \in X$, se tiene que $\left\{x_{n}: n \in \omega\right\} \cup\{x\}$ es un conjunto cerrado. Notemos que para cualquier $x \in X$, el rango de la sucesión trivial $\left\langle x_{n}\right\rangle$, donde $x_{n}=x$ para cada $n \in \omega$, es un conjunto cerrado, esto es, todo SC-espacio es $T_{1}$.

Proposición 2.11. Sea $(X, \tau)$ un SC-espacio secuencial. Entonces, para cualquier refinamiento $\sigma \supsetneq \tau$, existe un subconjunto infinito cerrado y discreto en $(X, \sigma)$.

Demostración. Sea $\sigma$ un refinamiento de $\tau$ con $\sigma \supsetneq \tau$, entonces existe un subconjunto $C$ de $X$ que es $\sigma$-cerrado pero no es $\tau$-cerrado. Como $(X, \tau)$ es secuencial, existe un punto $p \in \operatorname{cl}_{\tau} C \backslash C$ y una sucesión $\left\langle x_{n}\right\rangle$ en $C$ tal que $\left\langle x_{n}\right\rangle \rightarrow p$. Entonces, $p$ es el único punto de acumulación de $\left\langle x_{n}\right\rangle$ ya que $(X, \tau)$ es un SC-espacio, esto es, el conjunto $\left\{x_{n}: n \in \omega\right\} \cup\{p\}$ es $\tau$-cerrado. Como $C$ es $\sigma$-cerrado y $p \in X \backslash C \in \sigma$, concluimos que el conjunto $\left\{x_{n}: n \in \omega\right\}$ es un subconjunto infinito cerrado y discreto en $(X, \sigma)$.

Corolario 2.12. Si $(X, \tau)$ es un $S C$-espacio secuencial y disperso con orden de dispersión 2. Entonces, cualquier topología estrictamente más fina que $\tau$ no es una topología DNC.

Demostración. Sea $\sigma$ un refinamiento de $\tau$ y $\left\{x_{n}: n \in \omega\right\}$ es el subconjunto infinito cerrado y discreto en $(X, \sigma)$ construido en la proposición anterior. Como $(X, \tau)$ es disperso, existe $I \subset X$ subconjunto denso de puntos aislados y debido a que $(X, \tau)$ tiene orden de dispersión 2 , entonces no existen sucesiones $\tau$-convergentes en $X \backslash I$, por lo tanto, sin perdida de generalidad podemos suponer que $\left\{x_{n}: n \in \omega\right\} \subset I$ y no tiene puntos de acumulación en $(X, \sigma)$, es decir, $\left\{x_{n}: n \in \omega\right\}$ es un subconjunto cerrado infinito de puntos aislados en $(X, \sigma)$. Por lo tanto $(X, \sigma)$ no es $D N C$.

Para un espacio topológico $(X, \tau)$ y un punto $p \in X$, denotamos como $(X \backslash\{p\}) \oplus\{p\}$ la topología generada por la subbase $\tau \cup\{p\}$.

Teorema 2.13 ([27]). Un espacio infinito densamente numerablemente compacto es maximal densamente numerablemente compacto si y sólo si es un $S C$-espacio de Fréchet y disperso con orden de dispersión 2.

Demostración. La suficiencia se sigue del Corolario 2.12. 
Para la necesidad. Supongamos ahora que $(X, \tau)$ es un espacio maximal $D N C$, por tanto disperso con orden de dispersion 2 (Teorema 2.10). Denotamos $I$ como el conjunto de todos los puntos aislados de $X$. Si existe un punto $p \in X \backslash I$ que no es el único punto limite de algún subconjunto infinito numerable de $I$, entonces $(X \backslash\{p\}) \oplus\{p\}$ es una topología $D N C$ más fuerte que $\tau$ sobre $X$, lo cual es una contradicción.

Por lo tanto, cada punto $x \in X \backslash I$ es el único punto de acumulación de algún subconjunto infinito numerable $I_{x}$ de $I$. Como cada subconjunto infinito de $I_{x}$ debe tener a $x$ como único punto de acumulación, entonces $I_{x}$ es una sucesión que converge a $x$.

Para probar que $(X, \tau)$ es Fréchet, si $A \subset I$ y $x \in \operatorname{cl}(A)$ no es el único punto de acumulación de algún subconjunto infinito de $A$, entonces todo subconjunto infinito de $A$ tiene un punto de acumulación en $X \backslash I$ distinto de $x$. Se sigue que $\operatorname{cl}(A) \backslash\{x\}$ es un subespacio $D N C$ y entonces la topología generada por la subbase

$$
\tau \cup\{X \backslash(\operatorname{cl}(A) \backslash\{x\})\},
$$

difiere de $\tau$ sólo en $x$, hace de $X$ un espacio $D N C$ y es estrictamente más fuerte que $\tau$, lo cual es una contradicción.

Por lo tanto, existe un subconjunto infinito numerable $I_{x} \subset A$ que tiene $x$ como único punto de acumulación y así $I_{x} \rightarrow x$.

Lema 2.14. Si un espacio topológico $X$ es densamente numerablemente compacto, entonces todo subconjunto cerrado regular de $X$ es densamente numerablemente compacto.

Demostración. Sea $A=\operatorname{cl}(\operatorname{int}(A))$ un subconjunto cerrado regular de $X$ y $D$ testifica que $(X, \tau)$ es $D N C$. Notemos que $A \cap D \neq \emptyset$ y $c l_{A}(A \cap D)=A$, sea $\left\langle x_{n}\right\rangle$ una sucesión en $D \cap A$, entonces $\left\langle x_{n}\right\rangle$ tiene un punto de acumulación $p \in X$, como $A$ es cerrado, concluimos que $p \in A$.

Por el Lema 2.14 y por el hecho de que un subconjunto de un espacio de Fréchet y disperso hereda ambas propiedades, podemos obtener el siguiente hecho:

Corolario 2.15. Un subconjunto cerrado regular de un espacio maximal densamente numerablemente compacto es maximal densamente numerablemente compacto. 
Corolario 2.16. Si $X$ es un espacio maximal densamente numerablemente compacto, entonces todo subespacio densamente numerablemente compacto de $X$ es cerrado en $X$.

Demostración. Supongamos que $A \subset X$ es un subespacio $D N C$ de $X$ que no es cerrado. Como $X$ es maximal $D N C$, por el Teorema 2.13, $A$ hereda las propiedades de ser un SC-espacio infinito de Fréchet y disperso con orden de dispersión 2. Por estas propiedades, existe una sucesión inyectiva, por tanto un conjunto infinito $I$ de puntos aislados en $A$ sin punto de acumulación en $A$. Como $I$ está contenido en cualquier subconjunto denso de $A$, obtenemos el resultado.

Por los Teoremas 1.5 y 2.3, podemos concluir

Corolario 2.17. Un espacio densamente numerablemente compacto es maximal densamente numerablemente compacto si y sólo si es maximal tenuemente compacto.

Existen ejemplos de espacios maximales pseudocompactos y maximales tenuemente compactos que no son Hausdorff. El siguiente ejemplo es una simplificación de una construcción de H. Tong.

Ejemplo 2.18 ([43]). Existe un espacio $T_{1}$ que es maximal densamente numerablemente compacto pero no Hausdorff.

Demostración. Sea $X=(\omega \times \omega) \cup\{a, b\}$, donde $a, b \notin \omega \times \omega$. Dotamos a $X$ con la topología $\tau$ como sigue:

1) Cada punto de $\omega \times \omega$ es abierto,

2) Las vecindades de $a$ son de la forma $\{a\} \cup(\{k \in \omega: k \geq n\} \times \omega)$, para algún $n \in \omega$.

3) Si $b \in V \in \tau$, entonces para cada $m \in \omega, V$ contiene un conjunto de la forma $\{m\} \times\left\{k \in \omega: k \geq n_{m}\right\}$ para algún $n_{m} \in \omega$.

Notemos que por la construcción de $X$, todos sus puntos unitarios son cerrados, es decir, $X$ es $T_{1}$. Pero, cualquier vecindad de $a$ se interseca con cualquier vecindad de $b$, por lo cual $X$ no es de Hausdorff.

Además, con esta topología, tenemos que si para algún conjunto $A \subset \omega \times \omega$ tal que $a \in \operatorname{cl}(A)$, por la definición de las vecindades de $a$, siempre podemos encontrar una sucesión de $A$ que converge a a (mismo caso si $b \in \operatorname{cl}(A)$ ), por lo tanto $(X, \tau)$ es de Fréchet. También, si $\left\langle a_{n}\right\rangle$ es una sucesión en $\omega \times \omega$ 
tal que $\left\langle a_{n}\right\rangle \rightarrow a$, podemos tomar una vecindad $V$ de $b$ de tal forma que no contenga ninguno de los elementos de $\left\langle a_{n}\right\rangle$ (mismo caso para $b$ ). Esto es, cualquier sucesión de puntos aislados que converge a a (respectivamente a $b$ ) no tiene a $b$ (respectivamente a $a$ ) como un punto de acumulación. Por lo cual, $X$ es un $S C$-espacio de Fréchet. Además, notemos que $\omega \times \omega$ testifica que $X$ es $D N C$, por lo tanto $X$ es maximal $D N C$ (Teorema 2.13).

Ahora, es de nuestro interés conocer para cuales topologías $D N C$ existe un refinamiento maximal $D N C$. Ya que, por ejemplo la topología de la extensión de Katětov de los enteros $\kappa \omega$ (vease 3.12.6 de [18]) no tiene ningún refinamiento que sea a la vez $D N C$ y Fréchet (Corolario 2.12).

Sin embargo, las siguientes topologías son ejemplos de topologías con refinamiento maximal $D N C$ (vease Teorema 2.13):

1) La topología del duplicado de Alexandroff (Ejemplo 3.1.26 de [18]) de un SC-espacio, numerablemente compacto y Fréchet.

2) Una topología cofinita sobre un conjunto infinito.

3) La topología de la compactificación por un punto de un SC-espacio en el cual todo subconjunto compacto es finito.

Esto nos lleva a preguntarnos: Si un espacio de Hausdorff $X$ que es $D N C$, tiene un refinamiento $D N C$ el cual es maximal $D N C$, entonces: $i X$ tiene un subespacio denso de puntos aislados? La Proposición 2.19 responde esta cuestión de manera negativa.

Un espacio topológico $(X, \tau)$ es llamado maximal si $\tau$ es maximal en la colección de todas las topologías $\sigma$ tal que $(X, \sigma)$ no tiene puntos aislados.

Proposición 2.19. Existe un espacio densamente numerablemente compacto de Hausdorff que es denso en sí y cuya topología puede ser refinada a una topología maximal densamente numerablemente compacta.

Demostración. Sea $(Y, \tau)$ el espacio de Tychonoff, numerable y maximal construido en ZFC en [15]. Sea $\mathcal{P}$ la familia de todos los subconjuntos infinitos cerrados y discretos de $Y$ y sea $\mathcal{A}$ una familia maximal casi ajena en $\mathcal{P}$. Para cada $A \in \mathcal{A}$, sea $x_{A}$ un punto que no pertenece a $Y$, de tal manera que si $A, B \in \mathcal{A}$ con $A \neq B$, entonces $x_{A} \neq x_{B}$ y sea $X=Y \cup\left\{x_{A}: A \in \mathcal{A}\right\}$, definimos una topología $\sigma$ sobre $X$ como sigue:

(1) $Y \in \sigma$ y $\sigma \mid Y=\tau$, 
(2) Una vecindad abierta de $x_{A}$ es cualquier subconjunto de la forma $U \cup\left\{x_{A}\right\}$, donde $U \in \tau$ y $A \backslash U$ es finito.

Probaremos que $X$ tiene las propiedades requeridas. Notemos primero que $X$ es un espacio de Hausdorff, esto es, si $A, B \in \mathcal{A}$, entonces $F=A \cap B$ es finito y los conjuntos $A \backslash F$ y $B \backslash F$ son conjuntos cerrados disjuntos en $Y$. Como $Y$ es normal, existen conjuntos disjuntos $U, V \in \tau$ tales que $A \backslash F \subset U$ y $B \backslash F \subset B$, entonces los conjuntos $\left\{x_{A}\right\} \cup U$ y $\left\{x_{B}\right\} \cup V$ son $\sigma$-vecindades disjuntas de $x_{A}$ y $x_{B}$ respectivamente. Si por otra parte, $y \in Y$ y $x_{A} \in X$, entonces por regularidad de $Y$, existen $\tau$-vecindades de $y$ y $A \backslash\{y\}$ que hacen dos $\sigma$-vecindades disjuntas de y y $x_{A}$.

Ahora, para mostrar que $X$ es un espacio $D N C$, supongamos primero que $C \subset Y$ es un conjunto infinito cerrado y discreto de $Y$, entonces existe algún $A \in \mathcal{A}$ tal que $C \cap A$ es infinito, por tanto $x_{A} \in c l_{\sigma}(C)$ y cualquier enumeración de $A \cap C$ es una sucesión que converge a $x_{A}$. Más aún, si $F$ es cualquier subconjunto infinito de $Y$, entonces existe un subconjunto infinito cerrado y discreto $C \subset F$, por lo cual $F$ tiene un punto de acumulación $p$ en $(X, \sigma)$ y otra vez existe una sucesión en $F$ que converge a $p$.

Finalmente, para mostrar que la topología $\sigma$ puede ser refinada a una topología maximal $D N C$, consideremos la topología $\mu$ sobre $X$ obtenida de la subbase $\sigma \cup\{\{y\}: y \in Y\}$. El espacio $(X, \mu)$ es de Hausdorff, disperso de orden de dispersión 2. Como para cada $A \in \mathcal{A}$, la traza sobre $Y$ del filtro de $\mu$-vecindades de $x_{A}$ coincide con la traza sobre $Y$ del filtro de $\sigma$-vecindades de $x_{A}$, se sigue que $(X, \mu)$ es de Fréchet $D N C$, entonces por el Teorema 2.13, tenemos que $(X, \mu)$ es un espacio maximal $D N C$.

También, tenemos el siguiente resultado:

Teorema 2.20 ([27]). La topología de un SC-espacio X que es densamente numerablemente compacto y Fréchet puede ser refinada a una topología maximal densamente numerablemente compacta si y sólo si X posee un subconjunto denso de puntos aislados.

Demostración. Supongamos que $(X, \tau)$ es un $S C$-espacio, densamente numerablemente compacto de Fréchet con un conjunto denso de puntos aislados $I$. Definimos una topología $\sigma$ sobre $X$ como sigue:

(1) $\sigma|I=\tau| I$,

(2) Una $\sigma$-vecindad de $x \in X \backslash I$ es cualquier subconjunto de la forma $(U \cap I) \cup\{x\}$, donde $U$ es una $\tau$-vecindad de $x$. 
Con esta topología, $X$ es SC-espacio, Fréchet y disperso con orden de dispersión 2. Por el Teorema 2.13, $X$ es maximal $D N C$.

Inversamente, supongamos que $(X, \tau)$ es un espacio $D N C$ cuya topología puede ser refinada a una topología $\sigma$ maximal $D N C$. Sea $I$ el conjunto denso de puntos aislados de $(X, \sigma)$ testigo de que este es $D N C$. Consideramos $I$ como un subespacio de $(X, \tau)$, entonces $I$ es denso en $(X, \tau)$ y si $(I, \tau)$ no es discreto, como $(X, \tau)$ es Fréchet, existe una sucesión inyectiva $\left\{x_{n}: n \in \omega\right\}$ que converge a $x \in I$. Por lo tanto, como $(X, \tau)$ es un SC-espacio, el conjunto infinito $\left\{x_{n}: n \in \omega\right\} \subset I$ tiene un único punto de acumulación en $(X, \tau)$ y no tiene punto de acumulación en $(X, \sigma)$, lo cual es una contradicción.

La suficiencia del teorema anterior también es válida para $S C$-espacios densamente numerablemente compactos y secuenciales. Sin embargo, la necesidad no es valida para esta clase de espacios:

Ejemplo 2.21 ([31]). Existe un espacio de Hausdorff densamente numerablemente compacto, secuencial y disperso cuya topología no se puede refinar a una topología maximal densamente numerablemente compacta.

Demostración. Sea $\mathcal{A}$ una familia maximal casi-ajena de subconjuntos de $\omega$ y sea $\mathcal{B}$ una familia maximal casi-ajena de subconjuntos numerables de $\mathcal{A}$. Sea $X=\omega \cup \mathcal{A} \cup \mathcal{B}$, dotado con la topología:

1) Los puntos de $\omega$ son aislados.

2) Una vecindad de un punto $A \in \mathcal{A}$ es de la forma $\{A\} \cup A \backslash F$, donde $F \subset \omega$ es un conjunto finito.

3) Si $b \in \mathcal{B}$, una vecindad de $b$ es de la forma $\{b\} \cup(b \backslash g) \cup \bigcup\left\{A \backslash F_{A}\right.$ : $A \in b \backslash g$, donde $g \subset \mathcal{A}$ y $F_{A} \subset \omega$ son conjuntos finitos.

El conjunto de puntos aislados $\omega$ es testigo de que $X$ es $D N C$. Si para algún $E \subset \omega$ tenemos que $c l(E) \cap \mathcal{B} \neq \emptyset$, entonces $c l(E) \cap \mathcal{B}$ es infinito, por lo cual no existe ninguna sucesión de $\omega$ que converge a algún punto de $\mathcal{B}$. Esto a su vez implica que no existe ninguna sucesión de $\omega$ que converge a algún punto de $\mathcal{B}$ en cualquier topología $\sigma \supset \tau$. Por lo tanto, si $(X, \sigma)$ fuese maximal $D N C$, entonces cada punto de $\mathcal{B}$ debería ser aislado y contenido en algún subconjunto denso que sea testigo de que $(X, \sigma)$ es $D N C$. Como $\mathcal{B}$ es infinito, cerrado y discreto en $(X, \tau)$, esto no es posible.

Lema 2.22. Todo ordinal con la topología de orden es numerablemente compacto si y sólo si es densamente numerablemente compacto. 
Demostración. Bastará probar la necesidad. Sea $\Gamma$ un ordinal con la topología de orden y supongamos que $\Gamma$ no es numerablemente compacto. Entonces, existe una sucesión de puntos aislados $I$ sin punto de acumulación, entonces cualquier subconjunto denso de $\Gamma$ debe contener a $I$, por lo tanto $\Gamma$ no puede ser $D N C$.

El siguiente resultado muestra que un espacio $D N C$ no tiene por qué ser secuencial para que tenga un refinamiento que sea maximal $D N C$.

Teorema 2.23 ([27]). La topología de orden de cada ordinal densamente numerablemente compacto es refinable a una topología maximal densamente numerablemente compacto.

Demostración. Sea $\Omega$ un ordinal $D N C$, por tanto es un ordinal numerablemente compacto y $\Gamma \subset \Omega$ denota el conjunto de ordinales límites con cofinalidad $\omega$. Definimos una topología $\sigma$ sobre $\Omega$ como sigue:

(1) Cada punto de $\Omega \backslash \Gamma$ es aislado en $(\Omega, \sigma)$,

(2) Si $\alpha \in \Gamma$, entonces una $\sigma$-vecindad de $\alpha$ es de la forma $\{\alpha\} \cup(U \backslash \Gamma)$. donde $U$ es una vecindad de $\alpha$ en la topología de orden.

El conjunto $\Omega \backslash \Gamma$ es denso en $(\Omega, \sigma)$ y $(\Omega, \sigma)$ es $D N C$, Fréchet, disperso de orden de dispersión 2, por lo tanto es maximal $D N C$ (Teorema 2.13).

\subsubsection{Espacios T-maximales Densamente Numerablemente Compactos}

Dada una propiedad topológica $\mathcal{P}$, un espacio de Tychonoff $X$ es $T$-maximal $\mathcal{P}$ si tiene $\mathcal{P}$ y ninguna topología de Tychonoff más fuerte en $X$ tiene $\mathcal{P}$.

En la clase de los espacios de Tychonoff, los espacios tenuemente compactos coinciden con los espacios pseudocompactos y por el Teorema 2.3 sabemos que un espacio con un subconjunto denso de puntos aislados es pseudocompacto si y sólo si este es $D N C$. Además, por el Corolario 2.17 tenemos que un espacio regular $D N C$ es maximal $D N C$ si y sólo es maximal tenuemente compacto.

J. R. Porter, R. M. Stephenson y R. G. Woods muestran en [33] que un espacio regular es maximal tenuemente compacto si y sólo si es homeomorfo a un espacio $\Psi(\mathcal{M})$ generalizado (es decir, si en el Ejemplo 2.1 tomamos una familia maximal casi-ajena $\mathcal{M}$ de subconjuntos infinitos de cualquier conjunto infinito y discreto $D$ ). 
Por todo lo anterior, se concluye que un espacio regular es maximal $D N C$ si y sólo si este es un $\Psi(\mathcal{M})$ generalizado. Más aún, un espacio $\Psi(\mathcal{M})$ generalizado es localmente compacto y por tanto Tychonoff. Por lo cual, obtenemos el siguiente hecho:

Teorema 2.24. Un espacio de Tychonoff es maximal densamente numerablemente compacto si y sólo si es un espacio $\Psi(\mathcal{M})$ generalizado, para alguna familia $\mathcal{M}$ de un espacio discreto $D$.

La situación es muy diferente cuando consideramos espacios que son maximales densamente numerablemente compacto en la clase de espacios de Tychonoff. El siguiente hecho es parte del folclor (una prueba se puede encontrar en el Teorema 3.8 en [5])

Teorema $2.25([5])$. Un espacio de Tychonoff pseudocompacto y primero numerable es T-maximal pseudocompacto.

Por el teorema anterior, podemos concluir que un espacio de Tychonoff $D N C$ primero numerable es $T$-maximal $D N C$. A modo de contraste, el Ejemplo 2.2 es un espacio de Tychonoff pseudocompacto y primero numerable que no es $D N C$. También, tenemos el siguiente ejemplo (Ejemplo 3.15 en [6]):

Ejemplo 2.26 (Reznichenko). Existe un espacio de Tychonoff pseudocompacto Fréchet que no es T-maximal pseudocompacto.

Demostración. Existe un compacto Fréchet $K$ con la propiedad de que existe un $p \in K$, tal que $\beta(K \backslash\{p\})=K$ y entonces $K$ es maximal numerablemente compacto pero no $T$-maximal pseudocompacto, ya que la topología ( $K \backslash$ $\{p\}) \oplus\{p\}$ es pseudocompacta.

Para una mejor descripción del ejemplo anterior, véase la sección 8.4 de [19].

Notemos que en el ejemplo anterior, si $Y=K \backslash\{p\}$ y $D$ es algún denso en $Y$, entonces existe una sucesión en $D$ que converge a $p$ y esta sucesión no tiene punto de acumulación en $Y$, por lo cual $Y$ no es $D N C$. Por otra parte, el punto de la compactificación de Alexandroff de un espacio $\Psi$ es secuencial, pero no es $T$-maximal $D N C$. Entonces, es natural la pregunta: ¿Es un espacio de Fréchet $D N C$ un espacio T-maximal $D N C$ ? Para contestar esta pregunta, primero notemos que un espacio $T$-maximal $D N C$ debe tener muchas sucesiones convergentes no triviales. 
Lema 2.27. Si $X$ es un espacio T-maximal densamente numerablemente compacto y $D$ un subconjunto denso testigo de esto, entonces todo punto de $X \backslash D$ es un punto límite de una sucesión en $D$.

Demostración. Si $p \in X \backslash D$ no es punto límite de una sucesión en $D$, entonces no es el único punto de acumulación de un subconjunto infinito de $D$. Por lo tanto, $(X \backslash\{p\}) \oplus\{p\}$ es una topología Tychonoff densamente numerablemente compacto estrictamente más fuerte sobre $X$.

El siguiente teorema es análogo al Corolario 2.12 :

Teorema 2.28 ([27]). Supongamos que un $S C$-espacio $(X, \tau)$ es Fréchet y que $\sigma$ es una topología regular sobre $X$ tal que $\sigma \supsetneq \tau$, entonces $(X, \sigma)$ no es un espacio densamente numerablemente compacto.

Demostración. Supongamos que $\sigma$ y $\tau$ difieren en algún punto $x \in X$, como $(X, \sigma)$ es regular, entonces existe una $\sigma$-vecindad $V$ que es $\sigma$-cerrada de $x$ que no es una $\tau$-vecindad de $x$. Supongamos que $D$ es un subconjunto denso de $(X, \sigma)$ y por tanto, también de $(X, \tau)$. Como $X \backslash V \in \sigma$, se sigue que $D \cap(X \backslash V)$ es $\sigma$-denso, por tanto $\tau$-denso en $X \backslash V$ y como $x \in c l_{\tau}(X \backslash V)$, existe una sucesión inyectiva en $D \cap(X \backslash V)$ que $\tau$-converge a $x$. Como $(X, \tau)$ es un $S C$-espacio, se sigue que $x$ es el único punto de acumulación de esta sucesión y entonces en la topología $\sigma$ esta sucesión en $D$ no tiene punto de acumulación en $X$.

Este último resultado implica que el Ejemplo de Reznichenko (Ejemplo 2.26 ) es un espacio $T$-maximal $D N C$ (pero no $T$-maximal pseudocompacto).

Corolario 2.29. Un espacio de Tychonoff densamente numerablemente compacto y Fréchet es T-maximal densamente numerablemente compacto.

Notemos que la implicación inversa de este último hecho no es cierto en $Z F C$ ya que el espacio $\delta \mathbb{N}$ construido bajo $\mathrm{CH}$ en [13] es un espacio de Hausdorff compacto que es $T$-maximal pseudocompacto y por tanto $T$ maximal $D N C$ pero no tiene estrechez numerable.

\subsection{Grupos Topológicos y Paratopológicos Densamente Numerablemente Compactos}

El Ejemplo 3 en [39] es un ejemplo de un grupo paratopológico Hausdorff, segundo numerable y densamente numerablemente compacto que no es grupo 
topológico. En este apartado, mostraremos algunas condiciones bajo las cuales un grupo paratopológico $D N C$ resulta ser grupo topológico. Notemos que la propiedad $D N C$ se preserva bajo imagenes continuas.

Diremos que el grupo paratopológico $(G, \tau)$ es $\omega$-precompacto si para cada $U \in \xi(e)$ existe un subconjunto numerable $F_{U} \subset G$ tal que $G=F_{U} U=$ $U F_{U}$. Si el conjunto $F_{U}$ es finito para cada $U \in \xi(e)$, diremos que $G$ es un grupo paratopológico precompacto.

Proposición 2.30. Sea $(G, \tau)$ un grupo paratopológico, entonces las siguientes afirmaciones son equivalentes:

(a) $G$ es un grupo topológico $D N C$,

(b) $(G, \tau)$ es $D N C$, de Baire $y\left(G, \tau^{-1} \vee \tau\right)$ es $\omega$-precompacto,

(c) $\left(\Delta_{G}, \tau^{-1} \times \tau\right)$ es $D N C$.

Demostración. (a) $\Longrightarrow$ (b) Como todo grupo topológico DNC es pseudocompacto, entonces $(G, \tau)$ es $\omega$-precompacto y de Baire (Proposición 3 en [39]).

$(\mathrm{b}) \Longrightarrow(\mathrm{c})$ Consideremos el mapeo identidad $i:\left(G, \tau \vee \tau^{-1}\right) \rightarrow(G, \tau)$ Como $\left(G, \tau \vee \tau^{-1}\right)$ es un grupo topológico y $(G, \tau)$ un grupo paratopológico de Baire, por el Teorema 2.3 en [2] tenemos que $(G, \tau)$ es un grupo topológico (i.e. $\left.\tau=\tau^{-1}\right)$. Entonces $\pi:(\Delta, \tau \times \tau) \rightarrow(G, \tau)$ es un isomorfismo topológico y $\left(\Delta_{G}, \tau^{-1} \times \tau\right)\left(\equiv\left(\Delta_{G}, \tau \times \tau\right)\right)$ es homeomorfo a $(G, \tau)$. Por lo tanto, $\left(\Delta_{G}, \tau^{-1} \times\right.$ $\tau)$ es $D N C$.

(c) $\Longrightarrow$ (a) Como $\left(\Delta_{G}, \tau^{-1} \times \tau\right)$ es grupo topológico $D N C$ (por tanto pseudocompacto), entonces $(G, \tau)$ es grupo topológico (Lema 2.3 en [2]). Dado que $\tau=\tau^{-1}$ y la proyección $\pi:\left(\Delta_{G}, \tau \times \tau\right) \rightarrow(G, \tau)$ es un homeomorfismo. Por lo tanto, $(G, \tau)$ es $D N C$.

Un grupo paratopológico $G$ es topológicamente periódico si para cada $x \in G$ y cada vecindad $U \in \xi(e)$, existe un $n \in \omega$ tal que $x^{n} \in U$.

Por la Proposición 5 en [39], sabemos que todo grupo paratopológico de Hausdorff $D N C$ y periódico es un grupo topológico. El siguiente ejemplo de T. Banakh (Ejemplo 1 en [39]) es un grupo paratopológico $T_{1}$ que es $D N C$ y periódico pero no es de Hausdorff (y por tanto no es grupo topológico).

Ejemplo 2.31. Existe un grupo paratopológico $T_{1}$ periódico $D N C$ que no es un grupo topológico. 
Demostración. Para cada $n \in \mathbb{N}$, se define $C_{n}=\{0,1, \ldots, n-1\}$ dotado con la topología discreta y la operación binaria $+^{\prime}$ definida como $x+^{\prime} y \equiv x+y(\bmod$ $n)$ para cada $x, y \in C_{n}$. Tomamos la suma directa $G=\oplus_{n=1}^{\infty} C_{n}$ y denotamos $\mathcal{F}=\{f: \omega \backslash\{0\} \rightarrow \omega \mid f$ es no decreciente y no acotada $\}$.

Sea $S$ el conjunto de sucesiónes de $G$ que cumplen con la propiedad: para cada $\left\langle x_{n}\right\rangle \in S$, existe un $m \geq 1$ tal que para todo $n>m$, se tiene que $x_{n}=0$ y $0<x_{m}<f(m)$. Para cada $f \in \mathcal{F}$ tomamos una vecindad de la forma $O_{f}=\{0\} \cup\{s: s \in S\}$. Entonces, el conjunto $\left\{O_{f}: f \in \mathcal{F}\right\}$ es una base del neutro $e$ de $G$ para una topología $T_{1}$ que hace de $G$ un grupo paratopológico con las propiedades requeridas.

Proposición 2.32. Para cada grupo paratopológico de Hausdorff $D N C(G, \tau)$, las siguientes condiciones son equivalentes:

(a) $(G, \tau)$ es un grupo topológico,

(b) Para cada $g \in G$, el conjunto $\left\{g^{n}: n \geq 1\right\}$ es finito ó contiene un punto de acumulación.

(c) $(G, \tau)$ es topologicamente periódico.

Demostración. (a) $\Longrightarrow$ (b) Sea $g \in G$ y consideremos el subgrupo $c l\left(\left\{g^{n}\right.\right.$ : $n \in \mathbb{Z}\})$. Si existe un punto aislado en $\left\{g^{n}: n \geq 1\right\}$, entonces el elemento neutro $e$ es aislado en $\operatorname{cl}\left(\left\{g^{n}: n \in \mathbb{Z}\right\}\right)$ y por lo tanto $c l\left(\left\{g^{n}: n \in \mathbb{Z}\right\}\right)$ es un subgrupo discreto de un grupo topológico $D N C$. Por el Teorema 1.4.23 en [8], $\left\{g^{n}: n \geq 1\right\}$ es finito.

Ahora, si el neutro no es un punto aislado en $\operatorname{cl}\left(\left\{g^{n}: n \in \mathbb{Z}\right\}\right)$, entonces toda vecindad $V \in \xi(e)$ contiene un punto $z \in \operatorname{cl}\left(\left\{g^{n}: n \in \mathbb{Z}\right\}\right)$. El resultado se sigue por el hecho de que $z$ está en la cerradura de $\left\{g^{n}: n \in \mathbb{Z}\right\}$.

(b) $\Longrightarrow$ (c) Se obtiene del Teorema 2.9 en [2].

(c) $\Longrightarrow$ (a) Es la Proposición 5 en [39]

Un espacio $X$ es llamado totalmente densamente numerablemente compacto (ó $\boldsymbol{T}$-DNC) si existe un subconjunto denso $D$ en $X$ tal que toda sucesión en $D$ contiene una subsucesión con cerradura compacta en $X$. Notemos que todo espacio totalmente densamente numerablemente compacto es $D N C$.

Sea $\beta \omega$ la compactificación de Stone-Čech de $\omega$. Por un argumento standar, podemos obtener dos subespacios densos ajenos $A_{1}$ y $A_{2}$ de $\beta \omega \backslash \omega$. El conjunto $X=\omega \cup A_{1}$ es un subconjunto denso de $\beta \omega$, $\omega$ es denso en $X$ y cualquier subconjunto infinito de $\omega$ tiene puntos de acumulación en $A_{1}$, por lo cual $X$ 
es $D N C$ pero ningún subconjunto infinito $I$ de $\omega$ es compacto en $X$, ya que $I$ tiene puntos de acumulación en $A_{2}$. Por lo tanto, $X$ es un espacio $D N C$ que no es $T-D N C$.

Otro ejemplo de un espacio $D N C$ que no es $T-D N C$ en la clase de espacios de Hausdorff es la extensión de Katětov $\kappa \omega$ de $\omega$ en el cual todo subconjunto compacto es finito.

Teorema 2.33. Sea $(G, \tau)$ un grupo paratopológico T-DNC y $D$ un subconjunto denso en $G$ testigo de este hecho. Si el conjunto $D^{-1}$ es denso en $(G, \tau)$, entonces $(G, \tau)$ es un grupo topológico.

Demostración. Por Proposición 2.30, bastará probar que $\left(\Delta_{G}, \tau^{-1} \times \tau\right)$ es $D N C$. Notemos que si $D$ es denso en $(G, \tau)$, entonces $D^{-1}$ es denso en $\left(G, \tau^{-1}\right)$ y $\left(\Delta_{G}, \tau^{-1} \times \tau\right) \cap\left(D^{-1} \times D\right)$ es denso en $\left(\Delta_{G}, \tau^{-1} \times \tau\right)$.

Sea $\left\langle\left(a_{n}, a_{n}\right)\right\rangle_{n \in \omega}$ una sucesión en $\left(\Delta_{G}, \tau^{-1} \times \tau\right) \cap\left(D^{-1} \times D\right)$ y $\left\langle a_{n_{k}}\right\rangle_{k \in \omega}$ una subsucesión de $\left\langle a_{n}\right\rangle_{n \in \omega}$ tal que $c l\left(\left\langle a_{n_{k}}\right\rangle_{k \in \omega}\right)$ es un subconjunto compacto de $(G, \tau)$, por lo cual $c l_{\tau^{-1}}\left(\left\langle a_{n_{k}}\right\rangle_{k \in \omega}\right)$ es un subconjunto compacto de $\left(G, \tau^{-1}\right)$. Entonces, la sucesión $\left\langle\left(a_{n_{k}}, a_{n_{k}}\right)\right\rangle_{k \in \omega} \subset c l_{\tau^{-1}}\left(\left\langle a_{n_{k}}\right\rangle_{k \in \omega}\right) \times c l_{\tau}\left(\left\langle a_{n_{k}}\right\rangle_{k \in \omega}\right)$ es una sucesión contenida en un conjunto compacto, por lo tanto tiene un punto de acumulación $(x, y) \in\left(G \times G, \tau^{-1} \times \tau\right)$. Como $(G, \tau)$ es $T_{1}$, entonces $\left(\Delta_{G}, \tau^{-1} \times\right.$ $\tau)$ es un conjunto cerrado en $\left(G \times G, \tau^{-1} \times \tau\right)$ (Lema 2.2 in [2]). Por lo tanto, $(x, y) \in\left(\Delta_{G}, \tau^{-1} \times \tau\right)$ (es decir, $\left.x=y\right)$ y $\left(\Delta_{G}, \tau^{-1} \times \tau\right) \cap\left(D^{-1} \times D\right)$ es el subconjunto denso que testifica que $\left(\Delta_{G}, \tau^{-1} \times \tau\right)$ es $D N C$. 


\section{Capítulo 3}

\section{Espacios Selectivamente Tenuemente Compactos}

En 1994, I. Protasov muestra en [35] que todo grupo topológico precompacto contiene un subconjunto discreto no cerrado. Basado en este resultado, Y. F. Ortíz-Castillo y S. García-Ferreira en [32] introducen el siguiente concepto:

Un espacio topológico $T_{1}(X, \tau)$ es selectivamente tenuemente compacto, si cumple la siguiente propiedad:

(Q) Para toda sucesión $\left\langle U_{n}\right\rangle$ de subconjuntos abiertos ajenos dos a dos no vacíos de $X$, existen $x_{n} \in U_{n}$ para todo $n \in \omega$, tales que el conjunto $\left\{x_{n}: n \in \omega\right\}$ no es cerrado.

En la clase de espacios de Tychonoff este concepto fue llamado fuertemente pseudocompacto en [32] y selectivamente pseudocompacto en [14]. Debido a la longitud del nombre de esta clase de espacios, los espacios selectivamente tenuemente compactos serán denotados como $\boldsymbol{S e l T C}$, principalmente en las demostraciones de los resultados a lo largo del texto.

Notemos que si $X$ es un espacio $\operatorname{SelTC}$, entonces dada una sucesión $\left\langle U_{n}\right\rangle$ de subconjuntos abiertos ajenos dos a dos no vacíos de $X$, existen $x_{n} \in U_{n}$ para todo $n \in \omega$, tales que el conjunto $\left\{x_{n}: n \in \omega\right\}$ no es cerrado, es decir, el conjunto $\left\{x_{n}: n \in \omega\right\}$ tiene un punto de acumulación $p \in X \backslash \bigcup\left\{U_{n}: n \in \omega\right\}$, el cual es un punto de acumulación de la sucesión $\left\langle U_{n}\right\rangle$. Por lo anterior, podemos concluir que todo espacio SelTC es tenuemente compacto. 
Teorema 3.1. Todo espacio de Hausdorff densamente numerablemente compacto es selectivamente tenuemente compacto.

Demostración. Sea $(X, \tau)$ un espacio $D N C$ y $D$ el subconjunto denso de $X$ testigo de este hecho. Sea $\left\langle U_{n}\right\rangle$ una sucesión de subconjunto abiertos no vacíos ajenos dos a dos de $X$. Tomamos $x_{n} \in U_{n} \cap D$ para cada $n \in \omega$, como $X$ es $D N C$, el conjunto $\left\{x_{n}: n \in \omega\right\}$ tiene un punto de acumulación $p \in X \backslash \bigcup\left\{U_{n}: n \in \omega\right\}$. Por lo tanto el conjunto $\left\{x_{n}: n \in \omega\right\}$ no es cerrado.

Por el hecho anterior, tenemos que el espacio de Mrówka $(\Psi)$ (Ejemplo 2.1) es un espacio selectivamente tenuemente compacto que no es numerablemente compacto. También, D.B. Shakhmatov construye en [41] un ejemplo de un espacio pseudocompacto en el cual todos sus subconjuntos de cardinalidad menor que $\mathfrak{c}$ son cerrados, por tal motivo, dicho espacio no puede ser SelTC. Por lo anterior, concluimos que la clase de los espacios SelTC se encuentra entre la clase de los espacios numerablemente compactos y la clase de los espacios tenuemente compactos.

De aquí en adelante, los espacios selectivamente tenuemente compacto serán llamados Selectivamente pseudocompactos cuando nos encontremos en la clase de espacios de Tychonoff.

Consideremos ahora la siguiente propiedad:

$\left(\mathrm{Q}^{\prime}\right)$ Para toda sucesión $\left\langle U_{n}\right\rangle$ de subconjuntos abiertos no vacíos de $X$, existe un punto $p$ y para cada $n \in \omega$, un punto $x_{n} \in U_{n}$ tal que el conjunto $\left\{n \in \omega: x_{n} \in V\right\}$ es infinito para toda vecindad $V$ de $p$.

Al punto $p$ lo llamaremos $\boldsymbol{Q}$-punto crítico de la sucesión de conjuntos abiertos no vacíos $\left\langle U_{n}\right\rangle$ si existe un conjunto $\left\{x_{n}: n \in \omega\right\}$ tal que $x_{n} \in U_{n}$ para cada $n \in \omega$ y el conjunto $\left\{n \in \omega: x_{n} \in V\right\}$ es infinito para toda vecindad $V$ de $p$. Notemos que la propiedad $\left(\mathrm{Q}^{\prime}\right)$ implica la propiedad (Q) en la clase de espacios $T_{1}$.

En el Teorema 2.1 de [14] se mostró que la propiedad (Q) es equivalente a la propiedad $\left(\mathrm{Q}^{\prime}\right)$ en la clase de espacios de Tychonoff (el mismo argumento de la demostración muestra que coinciden en la clase de espacios $T_{3}$ ). Una pregunta inmediata es saber si son equivalentes en una clase más grande de espacios. Abordamos este problema a continuación. 
Teorema 3.2. Sea $X$ un espacio topológico $T_{1}$. Si $X$ posee un subconjunto denso $D$ tal que $\chi(D)=\omega$, entonces las propiedades $(Q)$ y $\left(Q^{\prime}\right)$ son equivalentes.

Demostración. Sólo necesitamos probar que la propiedad (Q) implica la propiedad $\left(\mathrm{Q}^{\prime}\right)$. Sea $\left\langle U_{n}\right\rangle$ una sucesión de subconjuntos abiertos no vacíos de $X$ y sea $D$ un subconjunto denso de $X$ en el cual cada punto tiene una base numerable de vecindades en $D$.

Si existe algún punto $y \in D$ tal que toda vecindad de $y$ interseca a un número infinito de elementos de $\left\langle U_{n}\right\rangle$, entonces tomamos una familia anidada de subconjuntos abiertos $\left\{V_{n}: n \in \omega\right\}$ en $X$ tal que $\left\{D \cap V_{n}: n \in \omega\right\}$ es una base local de $y$ en $D$. Entonces, existe algún elemento minimo $n_{0} \in \omega$ tal que $V_{0} \cap U_{n_{0}} \neq \emptyset$ y tomamos $x_{0} \in V_{0} \cap U_{n_{0}} \cap D$. Así, podemos tomar $n_{0}, n_{1}, \ldots, n_{m-1} \in \omega$ tal que $V_{k} \cap U_{n_{k}} \neq \emptyset$ y $x_{k} \in V_{k} \cap U_{n_{k}}$ para cada $k \leq m-1$. Sea $n_{m}$ el menor entero tal que $n_{m}>n_{m-1}$ y $V_{m} \cap U_{n_{m}} \neq \emptyset$ y tomamos $x_{m} \in V_{m} \cap U_{n_{m}} \cap D$. Entonces, la sucesión $\left\langle x_{n}\right\rangle$ converge a $y$ en $D$ (por tanto en $X$ ) y así $y$ es un Q-punto crítico de la sucesión $\left\langle U_{n}\right\rangle$ de subconjuntos abiertos.

Si por otra parte, para cada $y \in D$, existe alguna vecindad $W$ de $y$ que interseca sólo a un número finito de elementos de la sucesión $\left\langle U_{n}\right\rangle$, entonces tomamos $y_{0} \in U_{0} \cap D$ y tomamos un conjunto abierto $W_{0}$ tal que $y_{0} \in W_{0} \subset$ $U_{0}$ y $J_{0}=\left\{n \in \omega: W_{0} \cap U_{n} \neq \emptyset\right\}$ es finito. Tomamos $m_{1} \in \omega \backslash J_{0}$ y tomamos $y_{1} \in U_{m_{1}} \cap D$. Sea $W_{1}$ una vecindad abierta de $y_{1}$ tal que $y_{1} \in W_{1} \subset U_{m_{1}}$, $W_{1} \cap W_{0}=\emptyset$ y $J_{1}=\left\{n \in \omega: W_{1} \cap U_{n} \neq \emptyset\right\}$ es finito. Ya que tenemos seleccionados $0=m_{0}, m_{1}, \ldots, m_{n-1} \in \omega$, los puntos $y_{0}, \ldots, y_{n-1}$, subconjuntos finitos $J_{0}, \ldots, J_{n-1}$ de $\omega$ y los conjuntos abiertos $W_{0}, \ldots, W_{n-1}$, tomamos $m_{n} \in$ $\omega, y_{n} \in D$, un subconjunto abierto $W_{n}$ y un subconjunto finito $J_{n} \subset \omega$ como sigue:

(a) $m_{n} \in \omega \backslash \bigcup\left\{J_{k}: k \in\left\{m_{0}, m_{1}, \ldots, m_{n-1}\right\}\right\}$,

(b) $y_{n} \in U_{m_{n}} \cap D$,

(c) $W_{n}$ es una vecindad abierta de $y_{n}, W_{n} \subset U_{m_{n}}$ y $W_{n}$ interseca un número finito de elementos de la sucesión $\left\langle U_{n}\right\rangle$,

(d) $J_{n}=\left\{k \in \omega: W_{n} \cap U_{k} \neq \emptyset\right\}$.

Esta construcción recursiva, produce una sucesión $\left\langle W_{n}\right\rangle$ de subconjuntos abiertos no vacíos ajenos dos a dos tales que $W_{n} \subset U_{m_{n}}$ para cada $n \in \omega$. Aplicamos la propiedad (Q) a sucesión $\left\langle W_{n}\right\rangle$ y obtenemos un Q-punto crítico de la sucesión $\left\langle U_{n}\right\rangle$. Por lo cual, podemos concluir el resultado. 
Corolario 3.3. Si un espacio $T_{1}$ posee un subconjunto denso de puntos aislados, entonces las propiedades (Q) y ( $Q$ ') son equivalentes.

Teorema 3.4. Las propiedades $(Q)$ y $\left(Q^{\prime}\right)$ son equivalentes en la clase de espacios de Hausdorff.

Demostración. Supongamos que $X$ es un espacio de Hausdorff con la propiedad $(\mathrm{Q})$ y sea $\left\langle U_{n}\right\rangle$ una sucesión de subconjuntos abiertos no vacíos de $X$. Como $X$ es tenuemente compacto, la sucesión $\left\langle U_{n}\right\rangle$ tiene un punto de acumulación $q$.

(1) Si $\left\{n \in \omega: q \in U_{n}\right\}$ es infinito, entonces por elección $x_{n}=q$ para cada $n \in J$, por lo cual $q$ es un Q-punto crítico para la sucesión $\left\langle U_{n}\right\rangle$ y se cumple el resultado.

(2) Si $\left\{n \in \omega: q \in U_{n}\right\}$ es finito, entonces sin perdida de generalidad, asumimos que $q \notin U_{n}$ para todo $n \in \omega$. Sea $m_{0}=0$ y tomamos $y_{0} \in U_{0}$, como $y_{0} \neq q$ y $X$ es de Hausdorff, existen subconjuntos abiertos ajenos $V_{0}$ y $W_{0}$ con $y_{0} \in V_{0}$ y $q \in W_{0}$, asumimos que $V_{0} \subset U_{0}$.

Como $q$ es un punto de acumulación de la sucesión $\left\langle U_{n}\right\rangle$, existe un $m_{1} \neq$ $m_{0}$ tal que $W_{0} \cap U_{m_{1}} \neq \emptyset$, entonces podemos tomar $y_{1} \in W_{0} \cap U_{m_{1}}$. Otra vez, $y_{1} \neq q$ y $X$ es de Hausdorff, existen subconjuntos abiertos ajenos $V_{1}$ y $W_{1}$ con $y_{1} \in V_{0}$ y $q \in W_{1}$, asumimos que $V_{1} \subset W_{0} \cap U_{m_{1}}$ y $W_{1} \subset W_{0}$, así $V_{0}$ y $V_{1}$ son disjuntos. Nuevamente, utilizando el hecho de que $q$ es un punto de acumulación de $\left\langle U_{n}\right\rangle$, existe algún $m_{2} \notin\left\{m_{0}, m_{1}\right\}$ tal que $W_{1} \cap U_{m_{2}} \neq \emptyset$ y entonces tomamos $y_{2} \in W_{1} \cap U_{m_{2}}$ y conjuntos abiertos ajenos $V_{2}$ y $W_{2}$ tales que $y_{m_{2}} \in V_{2}$ y $q \in W_{2}$, también con $W_{2} \subset W_{1}$ y $V_{2} \subset U_{m_{2}} \cap W_{1}$. Esta construcción recursiva produce un conjunto infinito $K=\left\{m_{n}: n \in \omega\right\}$ y una sucesión de conjuntos abiertos ajenos $\left\langle V_{n}\right\rangle$ con la propiedad de que $V_{k} \subset U_{k}$ para cada $k \in K$. Por lo tanto, cualquier Q-punto crítico de la sucesión $\left\langle V_{n}\right\rangle$ es un Q-punto crítico de la sucesión $\left\langle U_{n}\right\rangle$.

El siguiente ejemplo, muestra que las propiedades $(\mathrm{Q})$ y $\left(\mathrm{Q}^{\prime}\right)$ no son equivalentes en la clase de espacios de $T_{1}$.

Ejemplo 3.5. Sea $\tau=\{(r, \infty) \backslash C: r \in \mathbb{R}$ y $C$ es numerable $\} \cup\{\emptyset, \mathbb{R}\}$. El espacio $(\mathbb{R}, \tau)$ tiene la propiedad $(Q)$ por vacuidad, ya que no existen conjuntos abiertos disjuntos no vacios. Pero la sucesión anidada de conjuntos abiertos $\mathcal{U}=\{(n, \infty): n \in \mathbb{N}\}$, no tiene Q-punto crítico. 
Es conocido el hecho de que todo espacio de Tychonoff puede ser encajado de manera densa en un espacio selectivamente pseudocompacto (la compactificación de Stone-Čech). Más aún, cada espacio de Hausdorff $X$ puede ser encajado como un subespacio abierto denso de su extensión de Katětov (Teorema 3.12 .6 de [18]), el cual es $H$-cerrado y por lo tanto tenuemente compacto, pero no necesariamente selectivamente tenuemente compacto.

Para un espacio de Hausdorff $X$, denotamos como $X_{0}$ el conjunto de puntos aislados de $X$ y $X_{1}=\operatorname{cl}\left(X \backslash \operatorname{cl}\left(X_{0}\right)\right)$.

Teorema 3.6. La extensión de Katětov $\kappa X$ de un espacio de Hausdorff $X$ es selectivamente tenuemente compacto si y sólo si $X_{1}$ es selectivamente tenuemente compacto.

Demostración. Para demostrar la suficiencia, notemos que $\kappa X$ es un espacio tenuemente compacto de Hausdorff y tales espacios con un denso de puntos aislados son SelTC (Teoremas 2.3 y 3.1).

Supongamos que $X_{1}$ es selectivamente tenuemente compacto y sea $\left\langle U_{n}\right\rangle$ una sucesión de conjuntos abiertos no vacíos ajenos dos a dos de $\kappa X$, sin perdida de generalidad, como $X$ es abierto y denso en $\kappa X$, asumimos que $U_{n} \subset X$ para cada $n \in \omega$. Entonces, $I=\left\{n \in \omega: U_{n} \cap \operatorname{cl}\left(X_{0}\right)\right\}$ es infinito ó $J=\left\{n \in \omega: U_{n} \cap\left(X \backslash c l\left(X_{0}\right)\right)\right\}$ es infinito. Si $I$ es infinito, el subconjunto cl $\left(X_{0}\right)$ es $S e l T C$ por lo antes mencionado, por lo cual existe un punto de acumulación de la sucesión $\left\langle U_{n} \cap X_{0}\right\rangle_{n \in I}$ en $\operatorname{cl}\left(X_{0}\right)$, el cual, es un Q-punto crítico de la sucesión $\left\langle U_{n}\right\rangle$ en $X$. Por otra parte, si $J$ es infinito, entonces como $U_{n} \cap\left(X \backslash \operatorname{cl}\left(X_{0}\right)\right) \supset U_{n} \cap X_{1}$, como $X_{1}$ es $S e l T C$, se obtiene el resultado.

Para demostrar la necesidad, supongamos que $X_{1}$ no es selectivamente tenuemente compacto, por lo cual existe $\left\langle V_{n}\right\rangle$ una sucesión de subconjuntos abiertos no vacíos ajenos dos a dos de $X_{1}$ que es disjunta de $X_{0}$ la cual no tiene Q-punto crítico en $X$. Procederemos a mostrar que $\left\langle V_{n}\right\rangle$ no tiene Qpunto crítico en $\kappa X$. Para esto, supongamos que para cada $n \in \omega, x_{n} \in V_{n}$. Como cada $V_{n}$ es infinito, se sigue que $V_{n} \backslash\left\{x_{n}\right\}$ es denso en $V_{n}$ y por tanto $\bigcup\left\{V_{n}: n \in \omega\right\}$ es miembro de algún ultrafiltro abierto $q$ sobre $X$, entonces también lo es $\bigcup\left\{V_{n} \backslash\left\{x_{n}\right\}: n \in \omega\right\}$. Pero esto significa que $q$ no es un punto de acumulación del conjunto $\left\{x_{n}: n \in \omega\right\}$ y por lo tanto, la prueba esta completa. 


\subsection{Espacios Maximales Selectivamente Tenuemente Compactos}

Nuestro primer objetivo es caracterizar a los espacios selectivamente tenuemente compactos en la clase de los espacios $T_{1}$. El primer párrafo de la prueba del siguiente resultado muestra que un espacio infinito $X$ maximal selectivamente tenuemente compacto debe poseer una familia infinita de subconjuntos abiertos no vacíos ajenos dos a dos de $X$.

Lema 3.7. Todo espacio maximal selectivamente tenuemente compacto tiene un punto aislado.

Demostración. Supongamos primero que $X$ no admite ninguna sucesión $\left\langle U_{n}\right\rangle$ de conjuntos abiertos no vacíos ajenos dos a dos. Si $p \in X$ no es aislado, entonces $X \backslash\{p\}$ tampoco admite tal sucesión infinita. Se sigue que $(X \backslash$ $\{p\}) \oplus\{p\}$ es una topología SelTC estrictamente más fuerte sobre $X$.

Supongamos que $(X, \tau)$ es $S e l T C$ sin puntos aislados. Sea $\left\langle U_{n}\right\rangle$ una sucesión de conjuntos abiertos no vacíos ajenos dos a dos de $X$ y para cada $n \in \omega$ tomamos $x_{n} \in U_{n}$ tal que $C=\left\{x_{n}: n \in \omega\right\}$ no es cerrado. Sea $\sigma$ la topología generada por la subbase $\tau \cup\{X \backslash C\}$. Entonces, tenemos que $C$ es denso en ninguna parte en $(X, \tau)$ y como los conjuntos abiertos en $(X, \sigma)$ son de la forma $U \cup(V \backslash C)$, donde $U, V \in \tau$, entonces $C$ es denso en ninguna parte en $(X, \sigma)$ también.

Ahora, mostraremos que $(X, \sigma)$ es una topología SelTC. Para esto, sea $\left\langle W_{n}\right\rangle$ una sucesión de conjuntos abiertos no vacíos ajenos dos a dos de $(X, \sigma)$. Como $C$ es denso en ninguna parte en $(X, \tau)$, se sigue que $W_{n} \backslash c l_{\tau}(C) \neq$ $\emptyset$, para cada $n \in \omega$. Entonces $\left\langle W_{n} \backslash c l_{\tau}(C)\right\rangle$ es una sucesión de conjuntos abiertos no vacíos ajenos dos a dos de $(X, \tau)$. Por lo tanto, para cada $n \in \omega$ podemos encontrar $z_{n} \in W_{n} \backslash c l_{\tau}(C)$ tal que el conjunto $\left\{z_{n}: n \in \omega\right\}$ no es $\tau$-cerrado y como $W_{n} \backslash \operatorname{cl}_{\tau}(C) \subset W_{n} \backslash C$, para cada $n \in \omega$, tenemos que $\left\{z_{n}: n \in \omega\right\}$ tampoco es $\sigma$-cerrado. Por lo cual, $(X, \sigma)$ es una topología SelTC estrictamente más fuerte que $(X, \tau)$, lo cual es una contradicción.

Lema 3.8. En un espacio maximal selectivamente tenuemente compacto todo subconjunto denso en ninguna parte es cerrado (y por lo tanto discreto).

Demostración. Notemos que en el primer párrafo del lema anterior se demostró que en un espacio maximal $S e l T C$, siempre existe una sucesión de conjuntos abiertos no vacíos ajenos dos a dos. 
Sean $(X, \tau)$ un espacio selectivamente tenuemente compacto, $A \subset X$ un subconjunto denso en ninguna parte no cerrado y $\sigma$ la topología generada por la subbase $\tau \cup\{X \backslash A\}$. Mostraremos que $(X, \sigma)$ es $\operatorname{SelTC}$. Supongamos que $\left\langle U_{n}\right\rangle$ es una sucesión de conjuntos abiertos no vacíos ajenos dos a dos en $(X, \sigma)$. Como $\operatorname{int}_{\tau}\left(c_{\tau}(A)\right)=\emptyset$, entonces $V_{n}=U_{n} \backslash c l_{\tau}(A) \neq \emptyset$ para cada $n \in$ $\omega$. Más aún, para cada $n \in \omega$, existen $S_{n}, T_{n} \in \tau$ tales que $U_{n}=S_{n} \cup\left(T_{n} \backslash A\right)$, por lo cual $V_{n}=\left(S_{n} \backslash c l_{\tau}(A)\right) \cup\left(T_{n} \backslash c l_{\tau}(A)\right) \in \tau$. Como $(X, \tau)$ es $\operatorname{SelTC}$, para cada $n \in \omega$, tomamos $x_{n} \in V_{n}$ tal que el conjunto $\left\{x_{n}: n \in \omega\right\}$ no es $\tau$ cerrado. Pero, $\left\{x_{n}: n \in \omega\right\}$ no es $\sigma$-cerrado, lo cual es una contradicción.

Corolario 3.9. Si un espacio maximal selectivamente tenuemente compacto tiene un subconjunto denso de puntos aislados, entonces es disperso de orden de dispersión 2.

Demostración. Sea $I$ el conjunto de puntos aislados de un espacio $X$ maximal SelTC. Entonces, todos los subconjuntos de $X \backslash I$ son densos en ninguna parte y por tanto cerrados. Por lo cual, $X \backslash I$ es cerrado y discreto.

Lema 3.10. Todo subconjunto cerrado regular de un espacio selectivamente tenuemente compacto es selectivamente tenuemente compacto.

Demostración. Sea $A=\operatorname{cl}(\operatorname{int}(A))$ un subconjunto cerrado regular de un espacio selectivamente tenuemente compacto $X$. Sea $\left\{U_{n} \cap A: n \in \omega\right\}$ una familia de conjuntos abiertos no vacíos ajenos dos a dos en $A$, donde $U_{n}$ es un conjunto abierto en $X$ para cada $n \in \omega$. Entonces, $\left\{U_{n} \cap \operatorname{int}(A): n \in \omega\right\}$ es una familia disjunta de conjuntos abiertos no vacíos en $X$. Como $X$ es $\operatorname{SelTC}$, existe $x_{n} \in U_{n} \cap \operatorname{int}(A)$ para cada $n \in \omega$, tal que el conjunto $\left\{x_{n}: n \in \omega\right\}$ no es cerrado en $X$, es decir, existe $x \in c l_{X}\left(\left\{x_{n}: n \in \omega\right\}\right) \backslash\left\{x_{n}: n \in \omega\right\}$. Como $A$ es cerrado, entonces $x \in A$. Por lo cual, $x \in \operatorname{cl}_{A}\left(\left\{x_{n}: n \in \omega\right\}\right) \backslash\left\{x_{n}: n \in \omega\right\}$, esto es, el conjunto $\left\{x_{n}: n \in \omega\right\}$ no es cerrado en $A$.

Teorema $3.11([1])$. Un subconjunto cerrado regular de un espacio maximal selectivamente tenuemente compacto es maximal selectivamente tenuemente compacto.

Demostración. Sea $C$ un subconjunto cerrado regular de un espacio maximal $\operatorname{SelTC}(X, \tau)$ y supongamos que $C$ no es un espacio maximal $S e l T C$. Entonces, existe una topología $\sigma$ sobre $C$ que es $\operatorname{SelTC}$ y estrictamente más fuerte que $\tau \mid C$. Por el Lema 3.8, tenemos que $\operatorname{fr}(C)$ es cerrado y discreto en $(X, \tau)$ 
y por lo cual $\sigma$ y $\tau$ coinciden en $\operatorname{fr}(C)$. Definimos una base $\mathcal{B}$ para una topología $\rho$ sobre $X$ como sigue:

Un conjunto $U \in \mathcal{B}$ si

1) Siempre que $x \in U \cap f r(C)$, entonces $\{x\}=U \cap f r(C)$ y

$$
U \cap\left(X \backslash \operatorname{int}_{\tau}(C)\right) \in \tau \mid\left(X \backslash \operatorname{int}_{\tau}(C)\right) \text { y } U \cap C \in \sigma,
$$

2) Si $U \cap f r(C)=\emptyset$, entonces $U \cap(X \backslash C) \in \tau$ y $U \cap C \in \sigma$.

Debido a que $\operatorname{int}_{\tau}(C)$ y $(X \backslash C)$ son dos $\tau$-abiertos cuya unión es densa en $(X, \tau)$, entonces cualquier sucesión infinita de abiertos no vacíos en $X$ interseca ya sea a $\operatorname{int}_{\tau}(C)$ ó $(X \backslash C)$ infinitamente. Por lo tanto, consideramos dos casos:

Sea $\left\langle U_{n}\right\rangle$ una sucesión de conjuntos abiertos no vacíos ajenos dos a dos de $(X, \rho)$.

Caso 1: El conjunto $\left\{n \in \omega: U_{n} \cap \operatorname{int}_{\tau}(C)\right\}$ es infinito, entonces $\left\langle U_{n} \cap C\right\rangle$ es una sucesión de conjuntos abiertos no vacíos ajenos dos a dos en $(X, \sigma)$, por lo cual, existen $x_{i} \in U_{n} \cap C$ tal que el conjunto $\left\{x_{i}: n \in \omega\right\}$ no es $\rho$-cerrado.

Caso 2: El conjunto $\left\{n \in \omega: U_{n} \cap(X \backslash C)\right\}$ es infinito, entonces $\left\langle U_{n} \cap\right.$ $(X \backslash C)\rangle$ es una sucesión de conjuntos abiertos no vacíos ajenos dos a dos en $(X, \rho)$, por lo cual, existen $x_{i} \in U_{n} \cap(X \backslash C)$ tal que el conjunto $\left\{x_{i}: n \in \omega\right\}$ no es $\rho$-cerrado.

Por todo lo anterior, podemos concluir que el espacio $(X, \rho)$ es $\operatorname{SelTC}$ y $\rho$ es estrictamente más fuerte que $\tau$. Lo cual contradice nuestra hipótesis.

Por el Lema 3.7, sabemos que un espacio maximal selectivamente tenuemente compacto tiene un punto aislado. También, sabemos que tales espacios no pueden tener subespacios cerrados regulares densos en sí y por lo tanto, estos espacios tienen un subconjunto denso de puntos aislados; por el Corolario 3.9 , obtenemos el siguiente resultado:

Teorema $3.12([1])$. Un espacio maximal selectivamente tenuemente compacto es disperso de orden de dispersión 2.

Teorema 3.13 ([1]). Un espacio maximal selectivamente tenuemente compacto es un SC-espacio de Fréchet.

Demostración. Supongamos ahora que $X$ es un espacio maximal SelTC, entonces es disperso con orden de dispersion 2 (Teorema 3.12), por lo tanto, 
existe un conjunto denso de puntos aislados de $X$. Por el Teorema 2.3, $X$ es $D N C$ y la prueba de este hecho es la misma que el argumento del Teorema 2.13 .

Por los dos resultados anteriores y la Proposición 2.11, obtenemos el siguiente resultado:

Corolario 3.14. Un espacio selectivamente tenuemente compacto es maximal selectivamente tenuemente compacto si y sólo si es un SC-espacio de Fréchet, disperso de orden de dispersión 2.

Por los Teoremas 2.3 y 3.12 , concluimos:

Corolario 3.15. Un espacio selectivamente tenuemente compacto es maximal selectivamente tenuemente compacto si y sólo si es maximal densamente numerablemente compacto.

\subsubsection{Espacios T-maximales Selectivamente Pseudocompactos}

El Corolario 3.4 en [6] muestra que un espacio topológico que posee un subconjunto pseudocompacto denso propio no es $T$-maximal pseudocompacto. A continuación, presentamos un resultado análogo para espacios selectivamente pseudocompactos:

Teorema 3.16 ([1]). Si $Y$ es un espacio de Tychonoff selectivamente pseudocompacto que posee un subconjunto pseudocompacto denso propio $D$, entonces $Y$ no es T-maximal selectivamente pseudocompacto.

Demostración. Tomamos $p \in Y \backslash D$ y mostraremos que $(Y \backslash\{p\}) \oplus\{p\}$ es una topología selectivamente pseudocompacta más fuerte sobre $Y$. Supongamos que $\left\langle U_{n}\right\rangle$ es una sucesión de conjuntos abiertos no vacíos ajenos dos a dos de $Y$. Si $p$ fuese el único punto de acumulación de $\left\langle U_{n}\right\rangle$, entonces la sucesión $\left\langle U_{n} \cap D\right\rangle$ no puede tener algún punto de acumulación en $D$, contradiciendo el hecho de que $D$ es pseudocompacto.

Ahora, mostraremos que el subespacio $Y \backslash\{p\}$ de $Y$ es selectivamente pseudocompacto. Supongamos que $\left\langle V_{n}\right\rangle$ es una sucesión de conjuntos abiertos no vacíos ajenos dos a dos de $Y \backslash\{p\}$. Como la sucesión $\left\langle V_{n}\right\rangle$ no converge a $p$, existe una vecindad $W$ de $p$ en $Y$ tal que $J=\left\{n \in \omega: V_{n} \not \subset c l(W)\right\}$ es infinito y así $\left\langle V_{n} \backslash \operatorname{cl}(W)\right\rangle_{n \in J}$ es una sucesión infinita de conjuntos abiertos 
no vacíos. Como $Y$ es selectivamente pseudocompacto, para cada $n \in J$, podemos encontrar $y_{n} \in V_{n} \backslash c l(W)$ tal que $\left\{y_{n}: n \in J\right\}$ no es cerrado en $Y$. Como $p$ no es un punto de acumulación de $\left\{y_{n}: n \in J\right\}$, se sigue que $\left\{y_{n}: n \in J\right\}$ no es cerrado en $Y \backslash\{p\}$.

Por lo tanto, $(Y \backslash\{p\}) \oplus\{p\}$ es una topología selectivalemente pseudocompacta más fuerte sobre $Y$ y así $Y$ no es $T$-maximal selectivamente pseudocompacto.

Corolario 3.17. El espacio de Reznichenko (Ejemplo 2.26) no es T-maximal selectivamente pseudocompacto.

\subsection{Grupos Topológicos y Paratopológicos Selectivamente Tenuemente Compactos}

Dado que la propiedad SelTC se preserva bajo funciones continuas (Proposición 3.1 en [14]), podemos obtener el siguiente resultado cuya demostración es análoga al argumento de la Proposición 2.30:

Proposición 3.18. Sea $(G, \tau)$ un grupo paratopológico, entonces las siguientes afirmaciones son equivalentes:

(a) $G$ es un grupo topológico SelTC,

(b) $(G, \tau)$ es SelTC de Baire y $\left(G, \tau^{-1} \vee \tau\right)$ es $\omega$-precompacto,

(c) $\left(\Delta_{G}, \tau^{-1} \times \tau\right)$ es SelTC.

Si queremos encontrar una condicion suficiente para la cual un grupo topológico pseudocompacto sea selectivamente pseudocompacto, necesitamos del siguiente concepto que fue introducido por Y. F. Ortíz-Castillo y S. García-Ferreira en [32]:

Un espacio topológico $X$ es $\boldsymbol{F}$-pseudocompacto, si para cada sucesión $\left\langle U_{n}\right\rangle$ de abiertos no vacíos ajenos dos a dos en $X$, existe un subconjunto discreto $D$ de $X$ contenido en $\bigcup\left\{U_{n}: n \in \omega\right\}$ tal que $c_{G}(D) \backslash \bigcup\left\{U_{n}: n \in\right.$ $\omega\} \neq \emptyset$ y $\left|D \cap U_{n}\right|<\omega$ para cada $n \in \omega$. Notemos que todo espacio SelFC es F-pseudocompacto.

Teorema 3.19 ([28]). Todo espacio topológico de Hausdorff $X$ que es $F$ pseudocompacto y secuencial es selectivamente tenuemente compacto. 
Demostración. Sea $\left\langle U_{n}\right\rangle$ una sucesión de abiertos no vacíos ajenos dos a dos en $X$. Como $X$ es un espacio F-pseudocompacto, existe un subconjunto discreto $D$ de $X$ contenido en $\bigcup\left\{U_{n}: n \in \omega\right\}$ tal que $c l_{G}(D) \backslash \bigcup\left\{U_{n}: n \in\right.$ $\omega\} \neq \emptyset$ y $\left|D \cap U_{n}\right|<\omega$, para cada $n \in \omega$. Debido a que $c l_{G}(D) \backslash \bigcup\left\{U_{n}\right.$ : $n \in \omega\} \neq \emptyset$ y $X$ es secuencial, existe $p \in \operatorname{cl}_{G}(D) \backslash D$ y una sucesión $S$ en $D \subset \bigcup\left\{U_{n}: n \in \omega\right\}$ tal que $S \rightarrow p$ y $\left|S \cap U_{n}\right|<\omega$ para cada $n \in \omega$.

Notemos que los puntos de $S$ son una subcolección de $D$, entonces $S$ puede no contener puntos de algunos $U_{n}$ 's. Entonces, para cada $i \in \omega$, tomamos

$$
x_{n}=\left\{\begin{array}{lll}
y_{n} \in S \cap U_{n}, & \text { si } & S \cap U_{n} \neq \emptyset \\
z_{n} \in U_{n}, & \text { si } & S \cap U_{n}=\emptyset .
\end{array}\right.
$$

Como la sucesión $\left\langle y_{n}\right\rangle$ es una subsucesión de $S$ que también converge a $p$, el conjunto discreto $\left\{x_{n}: n \in \omega\right\}=\left\{y_{n}: n \in \omega\right\} \cup\left\{z_{n}: n \in \omega\right\}$ no es cerrado y por tanto $G$ es $\operatorname{SelTC}$.

Por el Teorema 1.2 en [20], sabemos que todo grupo topológico pseudocompacto es F-pseudocompacto. Por el hecho anterior, podemos concluir el siguiente hecho:

Corolario 3.20. Todo grupo topológico pseudocompacto y secuencial es selectivamente pseudocompacto.

Un espacio topológico $X$ es selectivamente secuencialmente tenuemente compacto (ó $\boldsymbol{S S T C}$ ) si para cada sucesión $\left\langle U_{n}\right\rangle$ de subconjuntos abiertos no vacíos de $X$, existen $x_{n} \in U_{n}$ para cada $n \in \omega$ tal que la sucesión $\left\langle x_{n}\right\rangle$ tiene una subsucesión convergente. Estos espacios serán llamados selectivamente secuencialmente pseudocompactos cuando nos encontremos en la clase de espacios de Tychonoff.

Notemos que todo espacio $S S T C$ es SelTC. El ejemplo 5.7 de [14] muestra un grupo selectivamente pseudocompacto que no es selectivamente secuencialmente pseudocompacto.

Proposición 3.21. Todo espacio selectivamente tenuemente compacto de Hausdorff y secuencial es SSTC.

Demostración. Sea $\left\langle U_{n}\right\rangle$ una sucesión de subconjuntos abiertos no vacíos ajenos dos a dos de $X$, entonces existe $x_{n} \in U_{n}$ para cada $n \in \omega$ tal que el conjunto $\left\{x_{n}: n \in \omega\right\}$ no es cerrado, entonces existe $p \in \operatorname{cl}\left(\left\{x_{n}: n \in\right.\right.$ $\omega\}) \backslash\left\{x_{n}: n \in \omega\right\}$ y una sucesión de $\left\{x_{n}: n \in \omega\right\}$ que converge a $p$. 
Corolario 3.22. Sea G un grupo topológico secuencial. Entonces, son equivalentes:

(a) $G$ es pseudocompacto.

(b) $G$ es selectivamente pseudocompacto.

(c) $G$ es selectivamente secuencialmente pseudocompacto.

Demostración. (a) $\Longrightarrow$ (b) es el Corolario 3.20. (b) $\Longrightarrow$ (c) se cumple por la Proposición 3.21. (c) $\Longrightarrow$ (a) se cumple por definición de espacio selectivamente secuencialmente pseudocompacto. 


\section{Capítulo 4}

\section{Espacios Secuencialmente Tenuemente Compactos}

El siguiente concepto fue introducido en la clase de espacios de Tychonoff por G. Artico, U. Marconi, J. Pelant, L. Rotter y M.G. Tkachenko en [9]:

Un espacio topológico $(X, \tau)$ es secuencialmente tenuemente compacto, si cumple la siguiente propiedad:

(P) Para toda sucesión $\left\langle U_{n}\right\rangle$ de conjuntos abiertos no vacíos ajenos dos a dos de $X$, existe un subconjunto infinito $J \subset \omega$ y un punto $p \in X$ tal que el conjunto $\left\{n \in J: W \cap U_{n}=\emptyset\right\}$ es finito para toda vecindad $W$ de $p$.

En [9], este concepto aparece bajo el nombre de espacios secuencialmente pseudocompactos. Sin embargo, la definición de este concepto se le atribuye a E. A. Reznichenko en un manuscrito no publicado ([40]). Debido a la longitud del nombre de estos espacios, en ocasiones los espacios secuencialmente tenuemente compactos serán denotados como $\boldsymbol{S e c T C}$, principalmente en las demostraciones de los resultados en esta sección. De aquí en adelante, los espacios secuencialmente tenuemente compactos serán llamados secuencialmente pseudocompactos cuando nos encontremos en la clase de espacios de Tychonoff.

Notemos que si $\left\langle U_{n}\right\rangle$ es una sucesión de conjuntos abiertos no vacíos ajenos dos a dos en un espacio $X$ secuencialmente compacto, podemos tomar

$x_{n} \in U_{n}$ para cada $n \in \omega$ y la sucesión $\left\langle x_{n}\right\rangle$ tiene una subsucesión convergente a algún punto $p \in X$. Por lo cual, podemos concluir que la sucesión $\left\langle U_{n}\right\rangle \mathrm{y}$ el punto $p$ satisfacen la propiedad $(\mathrm{P})$. También, notemos que el punto $p$ 
descrito en la propiedad (P), en particular es un punto de acumulación para la familia dada de abiertos ajena dos a dos. Por lo anterior, podemos concluir que todo espacio secuencialmente compacto es $S e c T C$ y todo espacio $S e c T C$ es tenuemente compacto.

Teorema 4.1. Sea $X$ un espacio topológico que posee un subconjunto denso $D$ de puntos aislados. Entonces, $X$ es secuencialmente tenuemente compacto si y sólo si toda sucesión en $D$ contiene una subsucesión convergente.

Demostración. Sea $\left\langle x_{n}\right\rangle$ una sucesión en $D$ y tomamos un punto $p \in \operatorname{cl}\left(\left\langle x_{n}\right\rangle\right)$. Como $X$ es secuencialmente tenuemente compacto, existe $q \in X$ y $J \in[\omega]^{\omega}$ tal que $\left\{n \in J: x_{n} \notin V\right\}$ es finito, para toda vecindad $V$ de $q$. Por lo cual, la sucesión $\left\langle x_{n}\right\rangle_{n \in J}$ converge a $p$.

Ahora, sea $\left\langle U_{n}\right\rangle$ una sucesión de subconjuntos abiertos ajenos dos a dos no vacíos de $X$. Como $D$ es denso en $X$, tomamos $x_{n} \in D \cap U_{n}$ para cada $n \in \omega$, entonces la sucesión de puntos aislados $\left\langle x_{n}\right\rangle$ contiene una subsucesión $\left\langle x_{k}\right\rangle_{k \in \omega}$ que converge a un punto $q \in X$. Notemos que el conjunto infinito $I=\left\{k \in \omega: x_{k} \in U_{k}\right\}$ y el punto $q$ tienen la propiedad $(\mathrm{P})$.

La compactificación de Stone-Čech $\beta \omega$ de $\omega$, es un espacio compacto que no contiene sucesiónes convergentes no triviales (Corolario 3.6.15 en [18]). Por el Teorema 4.1 concluimos que $\beta \omega$ no es secuencialmente tenuemente compacto. También, S. García-Ferreira y A. H. Tomita muestran en [20] la existencia de un espacio secuencialmente pseudocompacto que no es secuencialmente compacto.

P. Lipparini demuestra en [25] que la propiedad (P) es equivalente a la siguiente propiedad en la clase de espacios topológicos:

$\left(\mathbf{P}^{\prime}\right)$ Para toda sucesión $\left\langle U_{n}\right\rangle$ de conjuntos abiertos no vacíos de $X$, existe un subconjunto infinito $J \subset \omega$ y un punto $p \in X$ tal que el conjunto $\{n \in$ $\left.J: W \cap U_{n}=\emptyset\right\}$ es finito para toda vecindad $W$ de $p$.

A este punto $p$ lo llamaremos $\boldsymbol{P}$-punto crítico de la sucesión de conjuntos abiertos $\left\langle U_{n}\right\rangle$. Un espacio con la propiedad $\left(\mathrm{P}^{\prime}\right)$ es llamado secuencialmente tenuemente compacto en [17].

El siguiente resultado es una ligera generalización del Teorema 2.2 (d) de [17].

Teorema 4.2 ([1]). Un espacio pseudoradial tenuemente compacto es secuencialmente compacto. 
Demostración. Supongamos que $\left\langle U_{n}\right\rangle$ es una sucesión de conjuntos abiertos no vacíos ajenos dos a dos de un espacio pseudoradial tenuemente compacto $X$. Si existe algún $q \in X$ tal que $J=\left\{n \in \omega: q \in \operatorname{cl}\left(U_{n}\right)\right\}$ es infinito, entonces obtenemos el resultado. Por otra parte, si no existe tal punto, existe un punto de acumualción $p$ de la sucesión $\left\langle U_{n}\right\rangle$ tal que $\left\{n \in \omega: p \in \operatorname{cl}\left(U_{n}\right)\right\}$ es finito y sin perdida de generalidad, asumimos que $p \notin c l\left(U_{n}\right)$ para todo $n \in \omega$ y por tanto $A=\bigcup\left\{\operatorname{cl}\left(U_{n}\right): n \in \omega\right\}$ no es cerrado. Como $X$ es pseudoradial, existe algún cardinal $\kappa$ y una $\kappa$-sucesión $T=\left\langle x_{\alpha}\right\rangle_{\alpha<\kappa}$ en $A$ que converge a $q \in X \backslash A$. Por lo tanto, existe $\alpha_{0}<\kappa$ tal que para cada $\alpha>\alpha_{0}$, tenemos que $x_{\alpha} \in X \backslash \operatorname{cl}\left(U_{n}\right)$ y se sigue que $\kappa$ tiene cofinalidad numerable y entonces existe una sucesión $S$ en $A$ que converge a $q$. Por lo cual se sigue que $X$ es $\operatorname{SecTC}$.

Debido a que $\beta \omega$ es un espacio de Hausdorff compacto no $S e c T C$, surge la cuestión de saber cuándo la compactificación de Stone-Čech de un espacio de Tychonoff ó la extención de Katětov de un espacio de Hausdorff es SecTC. Notemos que si un espacio de Hausdorff (ó Tychonoff) $X$ es SecTC, entonces $\kappa X$ (ó $\beta X$ ) es SecTC (inciso (c) del Teorema 3.1 en [17]). Consideremos entonces el problema inverso en el siguiente resultado.

Teorema 4.3. Sea $(X, \tau)$ un espacio topológico de Hausdorff. Entonces, $\kappa X$ es secuencialmente tenuemente compacto si y sólo si $X$ es secuencialmente tenuemente compacto.

Demostración. Como se mencionó anteriormente, la suficiencia se cumple. Probemos la necesidad suponiendo que $X$ no es SecTC. Entonces, existe alguna sucesión $\left\langle U_{n}\right\rangle$ de conjuntos abiertos no vacíos ajenos dos a dos de $X$ sin P-punto crítico en $X$. Por lo cual, si $p \in \kappa X \backslash X$, entonces una vecindad básica de $p$ es de la forma $U \cup\{p\}$ donde $U \in p$ y $U \subset X$. Para probar que $p$ no es un P-punto crítico de la sucesión $\left\langle U_{n}\right\rangle$, supongamos lo contrario, entonces existe alguna subsucesión $\left\langle U_{\phi(n)}\right\rangle$ tal que toda vecindad de $p$ interseca a todos excepto una cantidad finita de elementos de la subsucesión. Sin pérdida de generalidad, asumimos que $\phi(n)=n$ para cada $n \in \omega$.

Como $p$ es un ultrafiltro abierto sobre $X$, se sigue que $A=\bigcup\left\{U_{2 n}\right.$ : $n \in \omega\} \in p$ ó $B=\bigcup\left\{U_{2 n-1}: n \in \omega\right\} \in p$ pero no ambos. Por lo tanto, si $A \in p$, entonces $B \notin p$ y $p \notin c l(B)$, esto contradice la suposición de que toda vecindad de $p$ interseca a todos excepto un número finito de elementos de $\left\langle U_{n}\right\rangle$. 
Denotaremos como $\alpha X=X \cup\{\infty\}$ la compactificación en un punto de un espacio de Hausdorff $X$ localmente compacto.

Teorema 4.4 ([1]). Sea $X$ un espacio de Hausdorff localmente compacto $y$ pseudocompacto, entonces $\alpha X$ es secuencialmente pseudocompacto si y sólo si $X$ es secuencialmente pseudocompacto.

Demostración. Bastará mostrar la necesidad. Sea $\left\langle U_{n}\right\rangle$ una sucesión de conjuntos abiertos no vacíos ajenos dos a dos de $X$ sin $\mathrm{P}$-punto crítico en $X$, entonces $\infty$ es el único punto crítico de $\left\langle U_{n}\right\rangle$. Sin perdida de generalidad, asumimos que toda vecindad de $\infty$ interseca a todos excepto un número finito de elementos de $\left\langle U_{n}\right\rangle$.

Si la sucesión $\left\langle U_{n}\right\rangle$ no converge a $\infty$, entonces existe $V$ una vecindad cerrada de $\infty$ que no contiene una infinidad de subconjuntos $U_{n}$, esto es, $J=\left\{n \in \omega: U_{n} \backslash V \neq \emptyset\right\}$ es infinito. Pero entonces la sucesión $\left\langle U_{n} \backslash V\right\rangle$ es una sucesión de subconjuntos abiertos no vacíos ajenos dos a dos de $\alpha X$, la cual debe tener un P-punto crítico $p \in X$. Entonces $p$ es también un P-punto crítico de $\left\langle U_{n}\right\rangle$, lo cual es una contradicción.

Por lo tanto, no existe tal vecindad cerrada de $p$ y entonces $\left\langle U_{n}\right\rangle \rightarrow \infty$, lo cual muestra que $X$ no es pseudocompacto.

En el inciso (a) del Teorema 3.2 en [17] se muestra que toda imagen continua de un espacio secuencialmente pseudocompacto es un conjunto secuencialmente pseudocompacto. Por lo cual, tenemos el siguiente resultado:

Corolario 4.5. Si $X$ es localmente compacto y pseudocompacto, entonces $X$ tiene una compactificación secuencialmente pseudocompacta si y sólo si $X$ es secuencialmente pseudocompacto.

Demostración. Sabemos que si una compactificación $\nu X$ de $X$ es secuencialmente pseudocompacto, entonces $\alpha X$ también lo es (por ser imagen continua de $\nu X)$ y por el Teorema anterior obtenemos el resultado.

Es conocido el hecho de que si $\beta X$ es secuencial, entonces $X$ es pseudocompacto $([26])$. El siguiente resultado muestra que esto también es cierto si asumimos que $\beta X$ es secuencialmente pseudocompacto.

Teorema 4.6 ([1]). Si $\beta X$ es secuencialmente pseudocompacto, entonces $X$ es pseudocompacto. 
Demostración. Supongamos que $X$ no es pseudocompacto, entonces existe una familia discreta $\left\{U_{n}: n \in \omega\right\}$ de conjuntos abiertos no vacíos de $X$. Para cada $n \in \omega$ tomamos $x_{n} \in U_{n}$ y una función continua $f_{n}: X \rightarrow[0,1]$ tal que $f_{n}\left(x_{n}\right)=1$ y $f_{n}\left[X \backslash U_{n}\right]=0$.

Definimos $f=\sup \left\{f_{n}: n \in \omega\right\}$, notemos que $\operatorname{supp}(f) \subset \bigcup\left\{U_{n}: n \in \omega\right\}$. Sea $x \in X$ y $V$ vecindad abierta de $x$ en $X$, si $x \in U_{m}$ para algún $m \in \omega$, como $\left\{U_{n}: n \in \omega\right\}$ es una familia discreta, entonces $f_{n}(z)=0$ para todo $n \neq m$ y $f(z)=f_{m}(z)$ para todo $z \in V$, por lo cual $f$ es continua. Si $x \in X \backslash \bigcup\left\{U_{n}: n \in \omega\right\}$, tomamos $V$ vecindad abierta de $x$ en $X$, como $\left\{U_{n}: n \in \omega\right\}$ es una familia discreta, existe un único $k \in \omega$, tal que $V \cap U_{k} \neq \emptyset$ y por un argumento similar tenemos que $f$ es continua.

También, tenemos que $Z_{n}=f_{n}^{-1}\left[\frac{1}{2}, 1\right]$ es un conjunto nulo contenido en $U_{n}$ y es una vecindad de $x_{n}$ para cada $n \in \omega$, más aún, $Z=\bigcup\left\{Z_{n}: n \in \omega\right\}=$ $f^{-1}\left[\frac{1}{2}, 1\right]$ es un conjunto nulo. Sea $\mathcal{V}=\left\{\operatorname{int}_{X}\left(Z_{n}\right): n \in \omega\right\}$, entonces $\mathcal{V}$ es una familia discreta de conjuntos abiertos no vacíos de $X$ y mostraremos que la sucesión $\mathcal{S}=\left\{\operatorname{int}_{\beta X}\left(\operatorname{cl}_{\beta X}\left(\operatorname{int}_{X}\left(Z_{n}\right)\right)\right): n \in \omega\right\}$ de subconjuntos abiertos de $\beta X$ no tiene P-punto crítico en $\beta X$. Notemos primero que si los conjuntos:

$$
A=\operatorname{int}_{\beta X}\left(c_{\beta X}\left(\operatorname{int}_{X}\left(Z_{n_{1}}\right)\right)\right) \quad \text { y } \quad B=\operatorname{int}_{\beta X}\left(c_{\beta X}\left(\operatorname{int}_{X}\left(Z_{n_{2}}\right)\right)\right)
$$

son términos de la sucesión $\mathcal{S}$, entonces $c l_{\beta X}(A) \subset \operatorname{cl}_{\beta X}\left(Z_{n_{1}}\right)$ y $\operatorname{cl}_{\beta X}(B) \subset$ $c_{\beta X}\left(Z_{n_{2}}\right)$ y por tanto si $n_{1} \neq n_{2}$, entonces $A$ y $B$ son ajenos. También, si $p \in \beta X$ es un P-punto crítico de la sucesión $\mathcal{S}$, entonces $p \in \operatorname{cl}_{\beta X}(Z)$. Así como en el Teorema 4.4, debemos asumir que toda vecindad abierta de $p$ interseca a todos excepto una cantidad finita de elementos de $\mathcal{S}$ y por tanto todos excepto una cantidad finita de conjuntos $Z_{n}$. Por lo tanto, los conjuntos nulos $M=\bigcup\left\{Z_{2 n}: n \in \omega\right\}$ y $N=\bigcup\left\{Z_{2 n-1}: n \in \omega\right\}$ en $X$ tienen clausuras ajenas en $\beta X$ y entonces ni $p \notin c l_{\beta X}(M)$ ni $p \notin c l_{\beta X}(N)$, lo cual es una contradicción.

\subsection{Espacios Maximales Secuencialmente Tenuemente Compactos}

Recordemos que un espacio tenuemente compacto $X$ es $\boldsymbol{F C} \boldsymbol{C}$ si todo subespacio tenuemente compacto es cerrado en $X$. En [17] fue probado que un espacio $F C C$ es secuencialmente tenuemente compacto. Notemos que si $A$ es un subespacio tenuemente compacto de un espacio $(X, \sigma)$ y $\tau \subset \sigma$ entonces 
$(A, \tau)$ es tenuemente compacto y más aún, si $(X, \tau)$ es $F C C$ y $(X, \sigma)$ es tenuemente compacto, entonces también este último es $F C C$. Por Teorema 1.5 sabemos que todo espacio tenuemente compacto es maximal tenuemente compacto si y sólo si es $F C C$ y submaximal. Por lo cual, una submaximalización de un espacio FCC tenuemente compacto de Hausdorff es maximal SecTC.

Se sigue del Teorema 1.4 que un espacio maximal $S e c T C$ es $T_{1}$. También, por el Corolario 1.3, tenemos que un espacio submaximal SecTC donde todo subespacio SecTC es cerrado, es maximal SecTC.

Diremos que un espacio $S e c T C X$ es $\boldsymbol{S F C C}$ si todo subespacio $S e c T C$ es cerrado en $X$.

Teorema $4.7([1])$. Sea $(X, \tau)$ un espacio $T_{1}$ y SecTC. Entonces, una submaximalización de $X$ es SecTC (y por tanto una submaximalización de un espacio $T_{1}$, SFCC y SecTC es maximal SecTC).

Demostración. Supongamos que $\mathcal{F}$ es un filtro maximal de subespacios densos de $X$. La topología $\sigma$ generada por la subbase $\tau \cup \mathcal{F}$ es una topología submaximal en $X$. Necesitamos mostrar que $(X, \sigma)$ es $\operatorname{SecTC}$. Supongamos que $\left\langle U_{n}\right\rangle$ es una sucesión de conjuntos $\sigma$-abiertos no vacíos ajenos dos a dos. Cada conjunto $U_{n}$ podemos escribirlo como $U_{n}=V_{n} \cap F_{n}$, donde $V_{n} \in \tau$ y $F_{n} \in \mathcal{F}$. Entonces, como $(X, \tau)$ es $\operatorname{SecTC}$, existe un $p \in X$ y un conjunto infinito $S \subset \omega$ tal que toda $\tau$-vecindad $V$ de $p$ interseca a la familia $\left\{V_{n}: n \in\right.$ $S\}$ excepto un número finito de sus elementos. Pero, si $W$ es una $\sigma$-vecindad abierta de $p$, entonces $W=V \cap F$ para alguna $\tau$-vecindad abierta de $p$ y algún $F \in \mathcal{F}$ y entonces $\mathcal{F}$ es un filtro de $\tau$ conjuntos densos, se sigue que $W \cap U_{n}=V \cap F \cap V_{n} \cap F_{n}=\left(V \cap V_{n}\right) \cap\left(F \cap F_{n}\right) \neq \emptyset$ cuando $V \cap V_{n} \neq \emptyset$.

Notemos que si un espacio $(X, \tau) \operatorname{SecTC}$ tiene un subespacio $A$ que es $S e c T C$ y no cerrado, entonces para algún $p \in \operatorname{cl}(A) \backslash A$ definimos $\sigma$ como la topología generada por $\tau \cup\{X \backslash(\operatorname{cl}(A) \backslash\{p\})\}$, entonces $(X, \sigma)$ es $\operatorname{SecTC}$. Combinando esto y el Teorema anterior, obtenemos el siguiente resultado.

Corolario 4.8. Un espacio secuencialmente tenuemente compacto es maximal secuencialmente tenuemente compacto si y sólo si es submaximal y SFCC.

Incluso en la clase de los espacios de Tychonoff, un subespacio pseudocompacto cerrado de un espacio secuencialmente pseudocompacto no necesariamente es secuencialmente pseudocompacto. 
Ejemplo 4.9. Existe un espacio secuencialmente pseudocompacto que contiene un subespacio compacto que no es secuencialmente pseudocompacto.

Demostración. En [9] se probó que cada grupo topológico pseudocompacto es secuencialmente pseudocompacto, por lo cual el espacio $\{0,1\}^{\mathfrak{C}}$ es secuencialmente pseudocompacto. Pero, $\{0,1\}^{\mathfrak{c}}$ contiene una copia de $\beta \omega$ el cual no es secuencialmente pseudocompacto.

Como se ha mencionado, si un espacio $(X, \tau)$ es maximal SecTC, entonces es submaximal (Corolario 4.8), por tanto si $(X, \tau)$ tiene un conjunto denso $I$ de puntos aislados, entonces $(X, \tau)$ debe ser disperso de orden de dispersión 2. Por lo cual, si los elementos de la sucesión de conjuntos abiertos $\left\langle U_{n}\right\rangle$ en la definición de $\operatorname{SecTC}$ son puntos aislados, entonces existe un punto $p$ y una subsucesión $\left\langle U_{n}\right\rangle_{n \in J}$ que converge a $p$. Si para algún $A \subset I, p \in \operatorname{cl}(A) \backslash A$, pero $p$ no es el único punto de acumulación de algún subconjunto infinito de $A$, entonces la topología generada por $\tau \cup\{X \backslash(c l(A) \backslash\{p\})\}$ es $S e c T C$. Por tanto, si $p \in \operatorname{cl}(A)$, existe un conjunto de puntos aislados $I_{p} \subset A$ que tiene a $p$ como único punto de acumulación y entonces existe una sucesión en $I_{p}$ que converge a $p$. Entonces, obtenemos el siguiente resultado:

Teorema $4.10([1])$. Un espacio maximal secuencialmente tenuemente compacto que contiene un denso de puntos aislados es un SC-espacio de Fréchet.

\subsubsection{Espacios T-maximales Secuencialmente Pseudocompactos}

El espacio $\delta \mathbb{N}$ de [13] es T-maximal pseudocompacto (y por tanto Tmaximal secuencialmente pseudocompacto), secuencialmente compacto, radial pero no secuencial.

Un espacio $X$ es accesible de un subconjunto denso $D$ si cada punto de $X$ es el límite de una sucesión en $D$. Un espacio $X$ es fuertemente accesible de un subconjunto denso $D$ si siempre que $A \subset D$ y $x \in \operatorname{cl}(A)$, existe una sucesión de $A$ que converge a $x$. Una sucesión de conjuntos abiertos converge a $p \in X$ si toda vecindad de $p$ contiene todos excepto un número finito de elementos de la sucesión. La prueba del siguiente resultado, es similar a la prueba del Lema 2.8 de [3].

Teorema 4.11 ([1]). Si X es un espacio T-maximal secuencialmente pseudocompacto entonces es accesible de algún subespacio denso. Más aún, cada 
punto es el límite de una sucesión de conjuntos abiertos no vacíos ajenos dos a dos de $X$ y por tanto $\pi \chi(X)=\omega$ y si $X$ es regular, entonces $w(X) \leq 2^{c(X)}$. Demostración. Supongamos que $D$ es un subespacio denso de $X$ del cual $X$ no es accesible. Tomamos $p \in X \backslash D$ tal que ninguna sucesión de $D$ converge a $p$. Mostraremos que $(X \backslash\{p\}) \oplus\{p\}$ es una topología secuencialmente pseudocompacta más fuerte que $\tau$ y que es completamente regular siempre que $\tau$ es completamente regular. Con este fin, supongamos que $\left\langle U_{n}\right\rangle$ es una sucesión de conjuntos abiertos no vacíos ajenos dos a dos en $X$, si $p$ no es el único P-punto crítico de la sucesión $\left\langle U_{n}\right\rangle$, entonces existe $q \neq p$ el cual también es un P-punto crítico de $\left\langle U_{n}\right\rangle$. Como ninguna sucesión de $D$ converge a $p$, se sigue que la sucesión de conjuntos abiertos $\left\langle U_{n}\right\rangle$ no converge a $p$, entonces existe una vecindad cerrada $V$ de $p$ tal que $J=\left\{n \in \omega: U_{n} \backslash V \neq \emptyset\right\}$ es infinito y entonces $p$ no es un P-punto crítico de la sucesión $\mathcal{V}=\left\langle U_{n} \backslash V\right\rangle_{n \in J}$ de conjuntos abiertos no vacíos ajenos dos a dos. Por lo tanto, $\mathcal{V}$ debe tener un P-punto crítico $q$, el cual también es un punto crítico de $\left\langle U_{n}\right\rangle$.

Un argumento similar, prueba la segunda afirmación y la última desigualdad es una consecuencia del Teorema de Shapirovsky (Teorema 2.37 de [24]), el cual afirma que $w(X) \leq \pi \chi(X)^{c(X)}$ para espacios $T_{3}$.

Teorema 4.12 ([1]). Un espacio tenuemente compacto (respectivamente, pseudocompacto) que es fuertemente accesible de algún subconjunto denso primero numerable es secuencialmente tenuemente compacto (respectivamente, secuencialmente pseudocompacto).

Demostración. Sea $\left\langle U_{n}\right\rangle$ es una sucesión de conjuntos abiertos no vacíos ajenos dos a dos en $X$ con un subconjunto denso primero numerable $D$. Como $X$ es tenuemente compacto, la sucesión $\left\langle U_{n}\right\rangle$ tiene un punto de acumulación $p \in X$.

Si $p \in D$ entonces como $D$ es primero numerable y $p \in \operatorname{cl}_{D}\left(\bigcup\left\{U_{n} \cap D\right.\right.$ : $n \in \omega\})$, entonces tenemos que el conjunto $\left\{n \in \omega: p \in c l_{D}\left(U_{n} \cap D\right)\right\}$ ya sea finito ó infinito, $p$ es un P-punto crítico de la sucesión $\left\langle U_{n}\right\rangle$.

Si por otra parte, $p \in X \backslash D$ y $\left\{n \in \omega: p \in c l_{X}\left(U_{n} \cap D\right)\right\}$ es infinito, entonces $p$ es un P-punto crítico de $\left\langle U_{n}\right\rangle$. Si $\left\{n \in \omega: p \in c l_{X}\left(U_{n} \cap D\right)\right\}$ es finito, sin perdida de generalidad, asumimos que $p \notin c l_{X}\left(U_{n} \cap D\right)$ para todo $n \in \omega$, por lo tanto $A=\bigcup\left\{c l_{X}\left(U_{n} \cap D\right): n \in \omega\right\}$ no es cerrado. Como $X$ es fuertemente accesible de $D$, existe una sucesión $\left\langle x_{n}\right\rangle \subset D$ que converge a $p \in X \backslash A$. Entonces, existe $n_{0} \in \omega$ tal que para todo $n>n_{0}$ tenemos que $x_{n} \in X \backslash \operatorname{cl}\left(U_{n}\right)$ entonces existe una sucesión $S$ en $A$ que converge a $p$, por lo tanto $p$ es un P-punto crítico de $\left\langle U_{n}\right\rangle$. 
Tenemos el siguiente hecho (Teorema 2.4 de [3]):

Teorema 4.13 ([3]). Un espacio pseudocompacto que es fuertemente accesible de un subconjunto denso de puntos aislados es T-maximal pseudocompacto.

Se sigue el siguiente resultado:

Corolario 4.14. Un espacio pseudocompacto que es fuertemente accesible de un subconjunto de puntos aislados es T-maximal pseudocompacto y Tmaximal secuencialmente pseudocompacto.

\subsection{Grupos Topológicos Secuencialmente Tenuemente Compactos}

En [9] se mostró que los espacios tenuemente compactos y secuencialmente tenuemente compactos coinciden en la clase de grupos topológicos. Sin embargo, mostraremos que también coinciden en la clase de grupos paratopológicos de Hausdorff.

Lema 4.15 (Bourbaki, [11]). Si $A$ y $B$ son conjuntos abiertos ajenos en $(X, \tau)$, entonces $\operatorname{int}(c l(A))$ y $\operatorname{int}(\operatorname{cl}(B))$ son conjuntos abiertos ajenos en $\left(X, \tau_{S}\right)$ que contienen a $A$ y $B$ respectivamente.

El siguiente hecho muestra que la propiedad secuencialmente tenuemente compacta es semiregular:

Lema 4.16. Sea $X$ un espacio topológico. Entonces, $(X, \tau)$ es secuencialmente tenuemente compacto si y sólo si $\left(X, \tau_{S}\right)$ es secuencialmente tenuemente compacto.

Demostración. Debido a que $\tau_{S} \subset \tau$, se obtiene la suficiencia. Bastará probar la necesidad.

Supongamos que $\left(X, \tau_{S}\right)$ es secuencialmente tenuemente compacto y $(X, \tau)$ no lo es. Entonces existe una sucesión $\left\langle U_{n}\right\rangle$ de abiertos mutuamente ajenos no vacíos de $(X, \tau)$ de tal manera que para todo $x \in G$ y para todo $J \subset \omega$ infinito, existe $V \in \tau$ vecindad de $x$ tal que el conjunto $\left\{n \in J: V \cap U_{n}=\emptyset\right\}$ es infinito.

Por otra parte, por el Lema 4.15, tenemos que $\left\langle\operatorname{int}\left(\operatorname{cl}\left(U_{n}\right)\right)\right\rangle$ es una sucesión de abiertos mutuamente ajenos no vacíos de $\left(X, \tau_{S}\right)$ tal que $U_{n} \subset \operatorname{int}\left(\operatorname{cl}\left(U_{n}\right)\right)$ 
para todo $n \in \omega$. Como $\left(X, \tau_{S}\right)$ es secuencialmente tenuemente compacto, entonces existen $p$ y $J_{S} \subset \omega$ infinito tal que el conjunto $\left\{n \in J_{S}: \operatorname{int}(\operatorname{cl}(W)) \cap\right.$ $\left.\operatorname{int}\left(\operatorname{cl}\left(U_{n}\right)\right)=\emptyset\right\}$ es finito, para toda $\operatorname{int}(\operatorname{cl}(W))$ vecindad de $p$ en $\tau_{S}$.

Ahora, si tomamos $x=p$ y $J=J_{S}$, esto implica que existe $O \in \tau$ vecindad de $p$ tal que el conjunto $\left\{n \in J_{S}: O \cap U_{n}=\emptyset\right\}$ es infinito, esto es, $O \cap U_{n}=\emptyset$ para todo $n \in J_{S}$. Por Lema 4.15, tenemos que $\operatorname{int}(\operatorname{cl}(O))$ y $\operatorname{int}\left(\operatorname{cl}\left(U_{n}\right)\right)$ son conjuntos abiertos ajenos en $\left(X, \tau_{S}\right)$ que contienen a $O$ y $U_{n}$ respectivamente y $\operatorname{int}(\operatorname{cl}(O)) \cap \operatorname{int}\left(\operatorname{cl}\left(U_{n}\right)\right)=\emptyset$ para todo $n \in J_{S}$, es decir, el conjunto $\left\{n \in J_{S}: \operatorname{int}(\operatorname{cl}(O)) \cap \operatorname{int}\left(\operatorname{cl}\left(U_{n}\right)\right)=\emptyset\right\}$ es infinito y $p_{S} \subset O \subset \operatorname{int}(c l(O)) \in \tau_{S}$. Lo cual contradice el párrafo anterior.

Corolario 4.17 ([28]). Sea $(G, \tau)$ un grupo paratopológico de Haudorff. Entonces, $(G, \tau)$ es tenuemente compacto si y sólo si es secuencialmente tenuemente compacto.

Demostración. Bastará probar la necesidad. Supongamos que $(G, \tau)$ es tenuemente compacto, entonces $\left(G, \tau_{S}\right)$ es regular y tenuemente compacto, por tanto pseudocompacto, entonces $\left(G, \tau_{S}\right)$ es secuencialmente tenuemente compacto (Proposición 1.10 en [9]). Por el Lema 4.16, concluimos que $(G, \tau)$ es secuencialmente tenuemente compacto. 


\section{Capítulo 5}

\section{Conclusiones}

La presente tesis fue desarrollada en las áreas de topología general y álgebra topológica. Está basada principalmente en el estudio de las propiedades maximales que generalizan la compacidad y en el estudio de estas propiedades en la clase de grupos topológicos.

\subsection{Preguntas abiertas}

Las siguientes preguntas surgieron durante nuestra investigación y resultan de gran interés:

Problema 5.1. Caracterizar las topologías DNC, las cuales tienen un refinamiento maximal $D N C$.

Problema 5.2. Caracterizar los espacios T-maximales densamente numerablemente compactos.

Problema 5.3. ¿Existe un espacio X pseudocompacto no secuencialmente pseudocompacto tal que $\beta X$ es secuencialmente pseudocompacto?

Notemos que cada subespacio SecTC de $\kappa \omega$ es finito, pero $\kappa \omega$ no es $S e c T C$ ni $F C C$. Por lo cual, queda abierta la siguiente pregunta:

Problema 5.4. ¿Un espacio SFCC es FCC?

Problema 5.5. ¿Es cierto que $\beta X$ es secuencialmente pseudocompacto si $y$ sólo si $X$ es secuencialmente pseudocompacto? 
Sabemos por [33] que un espacio maximal tenuemente compacto es FCC y por el Teorema 2.2 de [17], tenemos que un espacio maximal tenuemente compacto es $S e c T C$. Como el espacio $\delta \mathbb{N}$ de [13] es T-maximal pseudocompacto pero no $F C C$, tenemos la siguiente pregunta:

Problema 5.6. ¿Es todo espacio T-maximal pseudocompacto, secuencialmente pseudocompacto? 


\section{Bibliografía}

[1] Alas, O. T., Martínez-Cadena, J. A. and Wilson, R. G., On sequential and selective feeble compactness, por aparecer en Houston Journal of Mathematics.

[2] Alas, O. T. and Sanchis, M. Countably compact paratopological groups, Semigroup Forum, 74 (2007), 423-438.

[3] Alas, O. T., Sanchis, M. and Wilson, R. G., Maximal pseudocompact and maximal R-closed spaces, Houston Journal of Mathematics, 38 No. 4 (2012), 1355-1367.

[4] Alas, O. T. and Wilson, R. G., Minimal properties between $T_{1}$ and $T_{2}$, Houston Journal of Mathematics, 32 No. 2 (2006), 493-504.

[5] Alas, O. T. and Wilson, R. G., The structure of the poset of regular topologies on a set, Applied General Topology, 12 No. 1 (2011), 1-13.

[6] Alas, O. T., Tkachuk, V. V. and Wilson, R. G., Maximal pseudocompact spaces and the Preiss-Simon property, Central European Journal of Mathematics, 12 No. 3 (2014), 500-509.

[7] Arhangel'skii, A. V. and Reznichenko, E. A. Paratopological and semitopological groups versus topological groups, Topology and its Applications, 151 (2005), pp. 107-119.

[8] Arhangel'skii, A. V. and Tkachenko, M. G. Topological Groups and Related Structures, Atlantis Studies in Mathematics, Vol.I, Atlantis Press and World Scientific, Paris-Amsterdan 2008.

[9] Artico, G., Marconi, U., Pelant, J., Rotter, L. and Tkachenko, M. G., Selections and suborderability, Fundamenta Mathematicae, 175 (2002), 1-33. 
[10] Berner, A. J., Spaces with dense conditionally compact subsets, Proceedings of the American Mathematical Society, 81 No. 1 (1981), 137-142.

[11] Bourbaki, N., Elements of Mathematics. General Topology. Part I. (Herman, Paris; Addison-Wesley, Reading, Mass., 1966).

[12] Cameron, D. E., A class of maximal topologies, Pacific Journal of Mathematics, 70 No. 1 (1977), 101-104.

[13] Devi, K. M., Meyer, P. R. and Rajagopalan, M., When does sequential compactness imply countable compactness?, General Topology and its Applications, 6 (1976), 279-289.

[14] Dorantes-Aldama, A. and Shakhmatov, D., Selective sequential pseudocompactness, Topology and its Applications, 222 (2017), 53-69.

[15] van Douwen, E., Applications of maximal topologies, Topology and its Applications, 51 (1993), 215-139.

[16] Dow, A., Compact $C$-closed spaces need not be sequential, Acta Mathematica Hungarica, 153 (2017), 1-15.

[17] Dow, A., Porter, J. R., Stephenson, R. M. and Woods, R. G., Spaces whose pseudocompact subspaces are closed subsets, Applied General Topology, 5 (2004), 243-264.

[18] Engelking, R., General Topology, Heldermann Verlag, Berlin, 1989.

[19] Fabian M., Gateaux Differentiability of Convex Functions and Topology, Canadian Mathematical Society Ser. Monogr. Adv. Texts, John Wiley and Sons, New York, 1997.

[20] García-Ferreira, S. and Tomita, A. H., A pseudocompact group which is not strongly pseudocompact, Topology and its Applications, 192 (2015) $138-144$.

[21] Gillman L. and Jerison M., Rings of Continuous Functions (Van Nostrand Reinhold, Princeton, NJ, 1960).

[22] Hewitt, E. A problem of set-theoretic topology, Duke Mathematical Journal, 10 (1943), 309-333. 
[23] Hewitt, E. Rings of Real-Valued Continuous Functions, I, Transactions of the American Mathematical Society, 64 (1948), 45-99.

[24] Juhász, I., Cardinal Functions in Topology - Ten Years Later, Mathematisch Centrum, Amsterdam, 1980.

[25] Lipparini, P., The equivalence of two definitions of sequential pseudocompactness, Applied General Topology, 17 No. 1 (2016), 1-5.

[26] Malykhin, V. I., Sequential bicompacta: Čech-Stone extensions and $\pi$ points, Moscow University Mathematics Bulletin, 30 (1975), 18-23

[27] Martínez-Cadena, J. A. and Wilson, R. G., Maximal densely countably compact topologies, Acta Mathematica Hungarica, 151 (2017), 259-270.

[28] Martínez-Cadena, J. A. and Sanchis, M., Some generalizations of compactness in topological groups, por aparecer en Topological Algebra and its Applications.

[29] Matveev, M., A survey on star covering properties, Topology Atlas preprint, April 15, 1998.

[30] Mrowka, S., On completely regular spaces, Fundamenta Mathematicae, 41, (1954) 105-106.

[31] Obersnel, F., Corrigendum to "Some notes on weakly Whyburn spaces", Topology and its Applications, 128 (2003), 257-262, Topology and its Applications, 138 (2004), 323-324.

[32] Ortíz-Castillo, Y. F. and García-Ferreira, S., Strong pseudocompact properties, Commentationes Mathematicae Universitatis Carolinae, 55 No. 1 (2014), 101-109.

[33] Porter, J. R., Stephenson, R. M. and Woods, R. G., Maximal feebly compact spaces, Topology and its Applications, 52 (1993), 203-219.

[34] Porter, J. R., Stephenson, R. M. and Woods, R. G., Maximal pseudocompact spaces. Commentationes Mathematicae Universitatis Carolinae, 35 No. 1 (1994), 127-145.

[35] Protasov I. V., Discrete subsets of topological groups, Mathematical Notes, Vol. 55, No. 1, (1994). 
[36] Raha, A. B., Maximal topologies, Journal of the Australian Mathematical Society, 15 (1973), 279-290.

[37] Ramanathan, A., Minimal-bicompact spaces, Journal of the Indian Mathematical Society, 19 (1948), 40-46.

[38] Ravsky, O. V., The topological and algebraic properties of paratopological groups, Ph.D. thesis. Lviv University, 2003 (in Ukrainian).

[39] Ravsky, O. V., Pseudocompact paratopological groups that are topological, eprint arXiv:1406.2001 (2014).

[40] Reznichenko, E. A., Embedding into first-countable pseudocompact spaces, unpublished manuscript.

[41] Shakhmatov, D. B., A pseudocompact Tychonoff space all countable subsets of which are closed and C-embedded, Topology and its Applications, 22 (1986) 139-144.

[42] Tkachuk, V. V. and Wilson, R.G., Maximal countably compact spaces and embeddings in MP-spaces, Acta Mathematica Hungarica, 145 No. 1, (2015), 191-204

[43] Tong, H., A note on minimal bicompact spaces (abstract), Bulletin of the American Mathematical Society 54 (1948), 478-479.

[44] Vaidyanathaswamy, R., Treatise on Set Topology, Indian Mathematical Society, Madras (1947). 
MAXIMALIDAD DE PROPIEDADES QUE GENERALIZAN LA COMPACIDAD

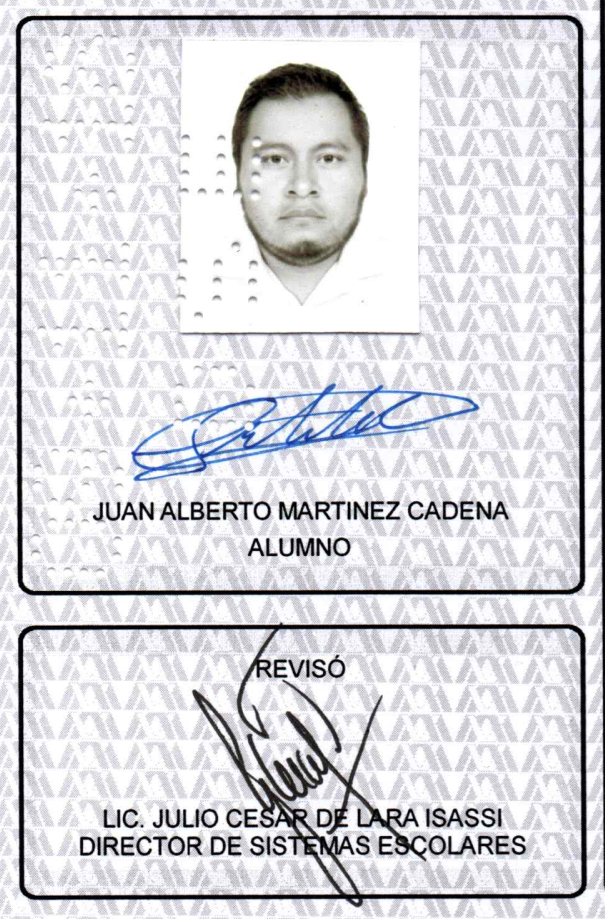

DIRECTOR DE LA DIVISIÓN DE CBI

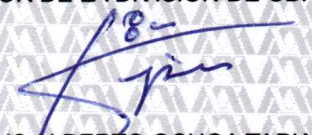
DR. JESUS ALBERTO OCHOA TAPIA
En la Ciudad de México, se presentaron a las 18:00 horas del día 5 del mes de noviembre del año 2018 en la Unidad Iztapalapa de la Universidad Autónoma Metropolitana, los suscritos miembros del jurado:

DR. RICHARD GORDON WILSON ROBERTS

DR. ANGEL TAMARIZ MASCARUA

DR. MANUEL SANCHIS LOPEZ

DR. SALVADOR GARCIA FERREIRA

DR. VLADIMIR TKATCHOUK VLADIMIROVICH

Bajo la presidencia del primero y con carácter de Secretario el último, se reunieron a la presentación de la Disertación Pública cuya denominación aparece al margen, para la obtención del grado de:

DOCTOR EN CIENCIAS (MATEMATICAS)

DE: JUAN ALBERTO MARTINEZ CADENA

y de acuerdo con el artículo 78 fracción IV del Reglamento de Estudios Superiores de la Universidad Autónoma Metropolitana, los miembros del jurado resolvieron:

\section{aprobar}

Acto continuo, el presidente del jurado comunicó al interesado el resultado de la evaluación $y$, en caso aprobatorio, le fue tomada la protesta.

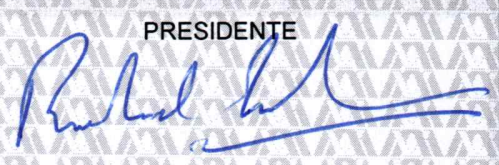

DR. RICHARD GORDON WILSON ROBERTS
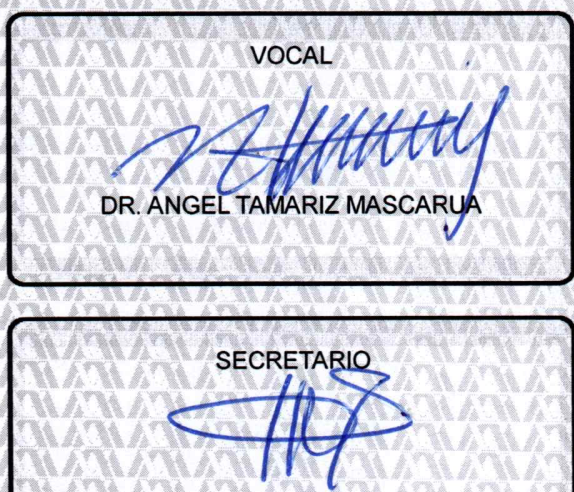

DR. VLADIMIR TKATCHOUK VLADIMIROVICH 